\title{
The Stability of Rotating Vortex Patches ${ }^{\star}$
}

\author{
Yieh-Hei Wan ${ }^{\star}$ \\ Department of Mathematics, State University of New York at Buffalo, Buffalo, New York 14214, USA
}

\begin{abstract}
In this paper we examine the nonlinear and linear stability of various rotating vortex patches. These patches include the Kirchhoff ellipse, the Kelvin waves, and the co-rotating uniform $m$ vortices. These are achieved by using relative variational methods and spectral analysis. Thus, we extend Arnol'd's idea for stability problems in $[1965,1969]$ to a non-smooth symmetric setting and also relate that to the usual linear stability analysis.
\end{abstract}

\section{Introduction}

Consider the motion of an incompressible flow with unit density in $R^{2}$ in the absence of external forces. At any instant, the velocity field $(u, v)=\left(\psi_{\bar{y}},-\psi_{\bar{x}}\right)$ for some stream function $\psi$ on $R^{2}=\{\mathbf{x}=(\bar{x}, \bar{y})\}$. The vorticity $\omega=v_{\bar{x}}-u_{\bar{y}}=$ $-\psi_{\bar{x} \bar{x}}-\psi_{\bar{y} \bar{y}}=-\Delta \psi$. We like to use the vorticity $\omega$ as the independent variable. Given $\omega$, let us choose a stream function $\psi=\int G \omega=(1 / 2 \pi) \int_{R^{2}} \omega\left(\overline{\mathbf{x}}^{\prime}\right) \ln \left(1 /\left|\overline{\mathbf{x}}^{\prime}-\overline{\mathbf{x}}\right|\right) d \mathbf{x}^{\prime}$, so that the velocity field is zero at infinity. The vorticity evolves according to the vorticity equation: $\omega_{t}+u \omega_{\bar{x}}+v \omega_{\bar{y}}=0$. Denote by $\Phi_{t}(\omega)$ the vorticity at time $t$, with initial vorticity $\omega$.

The energy $E$, the circulation $C$, the centre $\left(\bar{x}_{0}, \bar{y}_{0}\right)$, and the angular momentum $J$ are preserved under the motion $\Phi_{t}$. Recall that for a given vorticity $\quad \omega, \quad E=\frac{1}{2}\langle\omega, \psi\rangle=\frac{1}{2} \int_{R^{2}} \omega(\overline{\mathbf{x}}) \psi(\overline{\mathbf{x}}) d \overline{\mathbf{x}}, \quad C=\int_{R^{2}} \omega(\overline{\mathbf{x}}) d \overline{\mathbf{x}}, \quad \bar{x}_{0}=\int_{R^{2}} \bar{x} \omega(\overline{\mathbf{x}}) d \overline{\mathbf{x}}$, $\bar{y}_{0}=\int_{R^{2}} \bar{y} \omega(\overline{\mathbf{x}}) d \overline{\mathbf{x}}$, and $J=\int_{R^{2}}|\overline{\mathbf{x}}|^{2} \omega(\overline{\mathbf{x}}) d \overline{\mathbf{x}}$. A vortex patch $\omega$ is a vorticity in the form $\chi_{A}$, where $\chi_{A}$ stands for the characteristic function for a bound (measurable) set $A$ in $R^{2} \cdot \chi_{A_{j}}$ is called a component of $\chi_{A}$, if $A_{j}$ is a component of $A$. Vortex patches and their components are all preserved under the motion $\Phi_{t}$.

A vortex patch $\chi_{A}$ is said to be stationary if $\Phi_{t}\left(\chi_{A}\right)=\chi_{A}$ for all $t \geqq 0$. A vortex patch is said to be rotating if $\Phi_{t}\left(\chi_{A}\right)=\chi_{R_{\Omega t} A}$ for all $t \geqq 0$, where $R_{\theta}$ stands for a rotation through angle $\theta$. The Kirchhoff vortices $\chi_{E}$ (see Sect. 4) in which $E$ is an ellipse, are our model for rotating vortex patches. Two families of rotating vortex patches have been found recently. They are (a) the m-fold symmetric "Kelvin" waves

\footnotetext{
$\star \star$ Supported by National Science Foundation under grant DMS-8501746

$\star$ Dedicated to the memory of my grandmother, Lang-Chang Lee Wan
} 
$\mathscr{K}_{m}$ (see Deem and Zabusky [8], Burbea and Landau [7], Wu, Overman and Zabusky [33], etc.), (b) the co-rotating uniform $m$ vortices (see Dritschel [9].) Denote by $\mathscr{V}$ the space of all vortex patches with a $L^{1}$-topology induced from the $L^{1}$-norm on vorticities. Throughout this paper, our stationary or rotating patches will have $C^{1}$ boundaries.

Our basic problem in this paper is to examine the stability of rotating vortex patches from various viewpoints. More precisely, we aim to carry out theoretical investigations and concrete computations of the following topics:

(a) the $L^{1}$-stability via an energy method,

(b) the neutral stability (for a definition, see Sect. 3) through a spectral analysis,

(c) the relationship between the energy method and the spectral analysis.

Let us mention briefly (some of) of the literature which is closely related to our studies. Some general references are Lamb [17], Aref [2] and Zabusky [34]. In 1880, Kelvin ([17] p. 230) established the neutral stability of the circular patch in the plane. In 1887, Kelvin [15] proposed a variational principle for steady vortex motions. In particular, a steady vortex motion is stable if the energy reaches a maximum or minimum within a given vorticity and given moment of momentum at that vortex motion. Arnol'd [3,4] presented a method for proving a nonlinear version of the classical Rayleigh criterion for neutral stability of 2-dim shear flows. It involves a combination of a relative variational principle and convexity arguments.

Benjamin $[5,6]$, Turkington $[27,28]$ and others, established variational principles for steady vortex patches. Marsden and Weinstein [20] exploited Arnol'd's idea and put inviscid flows in a geometric setting through Poisson structures. Through this setting, Wan and Pulvirenti [30] established the $L^{1}$ stability of circular patches via either energy estimates or angular momentum estimates. See also Marchioro and Pulvirenti [19] for nonlinear stability of stationary vortex distributions.

In 1876, Kirchhoff $[17,18]$ found his elliptic vortex. It was proved by Love [18] that Kirchhoff vortex $\chi_{K}$ is neutrally stable iff its eccentricity $\leqq 2 \sqrt{2} / 3$. The $L^{1}$ stability for the same range, has just been established in Tang [26]. The rotating motion of two equal uniform vortices was studied by Saffman and Szeto [25]. The neutral stability of Kelvin waves and co-rotating uniform $m$ vortices have been analyzed by numerical methods in $[7,10]$ respectively.

Now, let us outline the general lines of our approach. It is rewarding to put the motion of incompressible inviscid flows in $R^{2}$ in a Hamiltonian setting. The sympletic leaf $M$ through a vortex patch $\omega_{0}$ is an isovortical surface, consisting of all isovortical variations of $\omega_{0}$ (cf. [20].) The nature action of rigid motions in $R^{2}$ leaves the leaf $M$ (a symplectic manifold), and the energy $E$ (a Hamiltonian) invariant. The angular momentum, centre of vorticities are the corresponding conserved quantities.

A rotating vortex patch will be regarded as a relative equilibrium with centre zero $\left(\bar{x}_{0}=\bar{y}_{0}=0\right)$ and the isotropy group $=$ the circle group $S^{1}$. For a non-stationary rotating patch $\chi_{A}$, the $S^{1}$ action is free near $\chi_{A}$ and the subspace $M_{0}=\{\omega \in M \mid J(\omega)$ $\left.=J\left(\chi_{A}\right), \bar{x}_{0}(\omega)=\bar{y}_{0}(\omega)=0\right\}$ is a $S^{1}$ invariant manifold. We expect that $\chi_{A}$ is a critical point of the energy function on $M_{0}$. Furthermore, we can verify the nonlinear stability of this rotating patch $\chi_{A}$ by establishing that the energy $E$ has a non- 
degenerate $S^{1}$-invariant local maximum at $\chi_{A}$ on $M_{0}$. The usual difficulty concerning the non-smoothness of the function $E$ in our setting can be overcome by techniques used for circular patches in [30] or elliptical patches in [26].

The commonly studied linearized equation possesses a Hamiltonian structure with the quadratic term of $\hat{E}=E+\frac{1}{2} \Omega \cdot J$ as a Hamiltonian. The existence of such a structure is suggested in Holm, etc. [14] Appendix A, and Abraham and Marsden [1] p. 252. From this fact, we are able to obtain our understanding of the linearized equation in a systematic way. In particular, they provide us, in both the theory and concrete examples, the direct connection between energy calculations for the nonlinear stability and the spectral analysis for the neutral stability. The separation lemma in Sect. 3 enables us to reduce this problem from an infinite dimensional into a finite dimensional one. The general idea of reduction for a linear Hamiltonian system has been used in Williamson [32] as a first step toward obtaining normal form for quadratic Hamiltonians. The relationship between stability and spectrum for a linear Hamiltonian system with periodic coefficients can be found in Krein [16].

Basic theorems about a relative variational principle, and a $L^{1}$-stability result for rotating patches are stated in Sect. 2 . We omit their proofs as they are basically a suitable abstraction of the argument in $[30,26]$. We derive the linearized equation in Sect. 3, and prove fundamental facts about this equation. It is shown that if the function $\hat{E}$ has an odd index, then the vortex patch must be unstable. To illustrate these basic results in Sects. 2 and 3, we apply them to the studies of Kirchhoff vortex patches in Sect. 4. The finite Kelvin waves $\mathscr{K}_{m}$ may be regarded as bifurcating from the circular patches. From this observation, the results and methods in Sects. 2 and 3 are applied to get information about nonlinear stability and neutral stability of these Kelvin waves near the circular ones in Sect. 5. The uniform $m$ vortices $\mathscr{T}_{m}$ are the desingularization of Thomson's $m$ vortices. After scaling, they can be regarded as a continuation of Thomson's $m$ vortices in a suitable sense. Again, using theorems and methods in Sects. 2 and 3, we obtain $L^{1}$-stability for a vortex pair $(m=2)$ in Sect. 6, and information about nonlinear stability and neutral stability for those uniform $m$ vortices $\mathscr{T}_{m}$ with small supports in Sect. 7. In order to make a smooth presentation, we put several technical propositions in the Appendices (A) through (D)

Finally, let us remark that our methods can also be applied to other relative equilibria, say with the real line $R$ as the isotropy group. The translating vortex pair [24], Hill spherical vortex [17], and Norbury's spherical-like vortex [23] belong to this category. For further information, please consult Wan [29].

\section{A Variational Principle and the Nonlinear Stability}

Following tradition, we also use an orthonormal frame $e_{x}, e_{y}$ which rotates around the origin with a fixed angular velocity $\Omega$ so that the coordinates are related by $\left(\begin{array}{c}\bar{x} \\ \bar{y}\end{array}\right)=\left(\begin{array}{cc}\cos \Omega t & -\sin \Omega t \\ \sin \Omega t & \cos \Omega t\end{array}\right)\left(\begin{array}{l}x \\ y\end{array}\right)(x=\bar{x}, y=\bar{y}$ at $t=0)$. In this frame, the relative velocity field $(u, v)=\left(\hat{\psi}_{y},-\hat{\psi}_{x}\right)\left(\equiv \hat{\psi}_{y} e_{x}-\hat{\psi}_{x} e_{y}\right)$ with $\hat{\psi}=\psi+\frac{1}{2} \Omega\left(x^{2}+y^{2}\right)$ the relative 
stream function. It is well known that a vortex patch $\omega=\chi_{A}$ is rotating with an angular velocity $\Omega$ iff $\hat{\psi}=$ constant (locally) on the boundary $\partial A$ of $A$.

Now, take a non-stationary rotating patch $\chi_{A}$, and notice its centre must be at the origin. This $S^{1}$ orbit $\chi_{R_{\theta} A}$ possesses a cross-section $\pi_{\alpha}=\left\{\omega \in \mathscr{V} \mid \int \alpha \omega=0\right\}$ at $\chi_{A}$ in the sense $(d / d \theta) \int \alpha \chi_{R_{\theta} A} \neq 0$ at $\theta=0$ for some smooth function $\alpha=\alpha(\bar{x}, \bar{y})$. Let $M$ be the "manifold" in $\mathscr{V}$ defined by $M=\{\omega \in \mathscr{V} \mid \omega$ has a fixed number of components with constant circulations $\}$. Denote by $M_{0}$ the "submanifold" in $M$ defined by constraints $J=$ constant, $\bar{x}_{0}=\bar{y}_{0}=0$. A rotating vortex is simply an extremum of $\hat{E}=E+\frac{1}{2} \Omega \cdot J$ on $M$ or on $M_{0}$. In order to obtain stability from variational primciples, one needs to establish on a priori estimate on $M_{0} \cap \pi_{\alpha}$ near $\chi_{A}$.

Fix a coordinate system $(\xi, \eta)$ in the $(x, y)$ plane, so that the above rotating patch $\chi_{A}$ is described by $\xi=\xi_{0}(\eta)$ for some smooth function $\xi_{0}$. Let $C_{1}, \ldots, C_{h}(\approx$ circles $)$ be the connected components of $\partial A$. Thus $\eta \in h$ copies of $S^{1}$. Set $Q=\left\{q \in L^{2}(\eta) \mid \int_{C_{1}} q d \eta\right.$ $\left.=\cdots=\int_{C_{h}} q d \eta=0\right\}$. Set $\mathcal{N}=\left\{q \in \mathscr{Q} \mid \int x q d \eta=\int y q d \eta=\int\left(x^{2}+y^{2}\right) q d \eta=\int \alpha q d \eta=0\right\}$. The linear spaces $\mathscr{Q}, \mathcal{N}$ can be regarded as the "tangent space" of $M$, and $M_{0} \cap \pi_{\alpha}$, respectively. Computations show that the "second order" term $\hat{E}_{2}$ of $\hat{E}=E+\frac{1}{2} \Omega \cdot J$ is given by $\left.\hat{E}_{2}=\frac{1}{2}\left[\int(\partial \hat{\psi} / \partial \xi) / I\right) q^{2}(\eta) d \eta+\iint G\left(\eta, \eta^{\prime}\right) q(\eta) q\left(\eta^{\prime}\right) d \eta d \eta^{\prime}\right]$. Here, $\partial \hat{\psi} / \partial \xi$, $I=\partial(x, y) / \partial(\xi, \eta), G$ are regarded as functions of $\eta\left(\eta\right.$ and $\left.\eta^{\prime}\right)$ by restricting to $\partial A$.

Now, we are ready to state our main results in this section.

Theorem 1. (A priori estimate) Suppose a non-stationary rotating vortex patch $\chi_{A}$ with an angular velocity $\Omega$ satisfies the following two conditions (1) $0<\Omega<1$, and $\left.\widehat{\psi}\right|_{\partial A}>\left.\hat{\psi}\right|_{\partial D}$ for some domain $D$ containing $A$. (2) the quadratic form $\hat{E}_{2}$ is negative definite on Hilbert space $\mathcal{N}$. Then, there exists a constant $c>0$, such that $\hat{E}\left(\chi_{A}\right)-\hat{E}\left(\chi_{B}\right)\left(=E\left(\chi_{A}\right)-E\left(\chi_{B}\right)\right) \geqq c\left|\chi_{A}-\chi_{B}\right|_{L^{1}}^{2}$ provided $\chi_{B} \in M_{0} \cap \pi_{\alpha}, \quad\left|\chi_{A}-\chi_{B}\right|_{L^{1}}$ small, and $B \subseteq D$.

Remark 1. The condition (1) insures that the values of $\hat{\psi}$ inside $A$ are larger than the values of $\hat{\psi}$ on $D \backslash A$. Thus, one can carry out the reduction process as that in $[30,26]$, for the non-stationary rotating vortex patch $\chi_{A}$.

Remark 2. It seems to the author the hypothesis in Theorem 1 are essentially necessary in order to reach the desired a priori estimate.

We shall say that a rotating vortex patch $\chi_{A}$ is $L^{1}$-stable relative to a disk $D$ centered at the origin (containing $A$ ) if given any $\varepsilon>0$, there exists $\delta>0$ such that, to each $t \geqq 0,\left|\Phi_{t}\left(\chi_{B}\right)-\Phi_{t^{\prime}}\left(\chi_{A}\right)\right|_{L^{1}}<\varepsilon$ for some $t^{\prime}$ provided $\left|\chi_{B}-\chi_{A}\right|_{L^{1}}<\delta$, support of $\Phi_{t}\left(\chi_{B}\right) \subseteq D$ for all $\bar{t}, 0 \leqq \bar{t} \leqq t$.

Using the established a priori estimate as that in $[30,26]$, we have

Theorem 2. Let $\chi_{A}$ be a non-stationary rotating vortex patch which fulfills conditions (1) and (2) in Theorem 1 , with $D$ a disk centered at the origin. Then $\chi_{A}$ is $L^{1}$-stable relative to the disk $D$.

\section{Linearization and Neutral Stability}

As before, we fix a coordinate system $(\xi, \eta)$ in the $(x, y)$ plane around a rotating vortex patch $\chi_{A}$, so that the boundary $\partial A$ of $A$ can be described as $\xi=\xi_{0}(\eta)$ for some $C^{1}$ function $\xi_{0}$. Denote by $\xi=\xi(\eta, t)$ the evolution of the boundaries of vortex patches 
$\chi_{B_{t}}$ near $\chi_{A}$ with $C\left(\chi_{B_{t}}\right)=C\left(\chi_{A}\right)$, as long as they remain radial. The normal component $I(\partial \xi / \partial t)$ of the velocity at the boundary $\partial B_{t}$ must be equal to the normal component $\partial \hat{\psi} / \partial \eta$ of the fluid velocity at the boundary. Thus, we obtain the evolution equation: $I(\partial \xi / \partial t)=(\partial \hat{\psi} / \partial \xi)(\partial \xi / \partial \eta)+(\partial \hat{\psi} / \partial \eta) ; \int_{\xi_{0}}^{\xi} I d \xi d \eta=0$. The linearized equation in $q$ $=I_{0} \delta \xi\left(I_{0}=I\left(\xi_{0}(\eta), \eta\right)\right)$ becomes

$$
\frac{\partial q}{\partial t}=(\mathscr{L} q)_{\eta}, \quad \text { with } \quad \mathscr{L} q=\left(\frac{\partial \hat{\psi}}{\partial \xi} / I_{0}\right) q+\int G q^{\prime} d \eta^{\prime}, \quad q \in Q .
$$

The linear space $Q$ has a weak symplectic structure $\omega\left(f_{\eta}, g_{\eta}\right)=\int f g_{\eta} d \eta$. (One can verify that this is the same weak symplectic structure as defined in [17] with $\psi_{1}=f$, $\psi_{2}=g$. See Corollary 1 of Appendix (A).) Notice $d \hat{E}_{2}(q)(\tilde{q})=\langle\mathscr{L} q, \tilde{q}\rangle$. Thus,

Theorem 3. (Linearization) The linearized equation $\partial q / \partial t=(\mathscr{L} q)_{\eta}$ on $Q$ is a Hamiltonian system with Hamiltonian system with Hamiltonian $\hat{E}_{2}=\frac{1}{2}\langle q, \mathscr{L} q\rangle$ the quadratic term of $\hat{E}$, and the weak symplectic structure $\omega\left(f_{\eta}, g_{\eta}\right)=\int f g_{\eta} d \eta$.

We shall say that the rotating vortex patch $\chi_{A}$ is neutrally stable if the linear transformation $(\mathscr{L} q)_{\eta}$ on $Q$ has only purely imaginary eigenvalues. Thus, $\chi_{A}$ is neutrally unstable (or instable) iff $(\mathscr{L} q)_{\eta}$ on $Q$ has an eigenvalue with positive real part. One expects neutral instability implies the $L^{1}$-instability.

The induced transformation of $(\mathscr{L} q)_{\eta}$ on $\mathscr{N}$ can be put in the form $(\mathscr{K} q)_{\eta}$ with $\mathscr{K} q=\mathscr{L} q+\left\langle\mathscr{L} q, \alpha_{\eta}\right\rangle\left(x^{2}+y^{2}\right)$. One verifies that $\dot{q}=(\mathscr{K} q)_{\eta}$ on $\mathscr{N}$ is again a Hamiltonian system with the Hamiltonian $\hat{E}_{2} \mid \mathcal{N}$ and the restricted symplectic structure from $Q$. By Propositions 1 and 2 in Appendix (A), we know that both $(\mathscr{L} q)_{\eta},(\mathscr{L} q)_{\eta}$ have discrete spectra and eigenvalues of $\left.(\mathscr{L} q)_{\eta}\right\}=\{0,0$, $\pm i \Omega\} \cup\left\{\right.$ eigenvalues of $\mathscr{K}_{\eta}$ on $\left.\mathscr{N}\right\}$. An operator is said to have discrete spectrum if its spectrum consists of isolated eigenvalues with finite multiplicities. From this fact, one can readily derive relationship between nonlinear stability and neutral stability. Immediately, one sees that if $\hat{E}_{2}$ has a non-degenerate local maximum then the system must be neutrally stable (by using $\hat{E}_{2}$ as a Lyapunov function).

Let us enclose a portion $\Sigma^{\prime}$ of the spectrum of $(\mathscr{K} q)_{\eta}$ on $\mathscr{N}$ by a simple curve $\Gamma$, symmetric with respective to $x$-axis and $y$-axis. The projection associated to $\Sigma^{\prime}$ is defined by $P_{\Sigma^{\prime}}=(-1 / 2 \pi i) \int_{\Gamma}\left(d \zeta / \mathscr{K}_{\eta}-\zeta\right)$.

Lemma 1. (a separation lemma) (a) $\mathscr{N}=P_{\Sigma^{\prime}}(\mathscr{N}) \oplus\left(I-P_{\Sigma^{\prime}}\right)(\mathcal{N})$ is a decomposition into symplectic subspaces, skew-orthogonal to each other.

(b) $\hat{E}_{2}\left(v_{1}+v_{2}\right)=\hat{E}_{2}\left(v_{1}\right)+\hat{E}_{2}\left(v_{2}\right)$ for $\left(v_{1}, v_{2}\right) \in P_{\Sigma^{\prime}}(\mathscr{N}) \oplus\left(I-P_{\Sigma^{\prime}}\right)(\mathscr{N})$.

Proof. To see (a), it suffices to observe $\omega\left(\mathscr{K}_{\eta} u, v\right)=\omega\left(u, \mathscr{K}_{\eta} v\right)$.

To see (b), it suffices to notice $\left\langle\mathscr{K} v_{1}, v_{2}\right\rangle=\omega\left(\left(\mathscr{K} v_{1}\right)_{\eta}, v_{2}\right)$. To each $\lambda \in \Sigma\left(\mathscr{K}_{\eta}\right)$ the spectrum of $\mathscr{K}_{\eta}$, set $N_{[\lambda]}=P_{[\lambda]}$ with $[\lambda]=\{\lambda, \bar{\lambda},-\lambda,-\bar{\lambda}\}$ (by Lemma 1 , (a), $\bar{\lambda},-\lambda$, $\left.-\bar{\lambda} \in \Sigma\left(\mathscr{K}_{\eta}\right)\right)$. We know that these symplectic sub-spaces are linearly independent and their linear combinations from a dense subspace of $\mathscr{N}$.

The index of $-\hat{E}_{2} \mid \mathcal{N}$ is equal to the dimension of maximal linear subspace on which $-\hat{E}_{2}$ is negative definite. The index of $-\hat{E}_{2} \mid \mathscr{N}$ is always finite. If $-\hat{E}_{2} \mid \mathcal{N}$ has an even index, the system can either be neutrally stable or not. (Such examples are given in Sects. 5 and 7. See Remarks 3 and 5.) However, for $-\hat{E}_{2} \mid \mathcal{N}$ being odd index we must have instability. 
Theorem 4. (Instability) Suppose $-\hat{E}_{2} \mid \mathcal{N}$ is non-degenerate and has an odd index. Then the linear transformation $(\mathscr{L} q)_{\eta}$ has an odd number of pairs of eigenvalues in the form $\pm \lambda, \lambda>0$.

Proof. Set $\Sigma^{*}=\left\{\lambda \in \Sigma\left(\mathscr{K}_{\eta}\right) \mid-\hat{E}_{2}\right.$ can assume negative values on $N_{[\lambda]}$ (defined after Lemma 1) $\}$. The number of elements in $\Sigma^{*}$, must be bounded by $4 \cdot$ index $\left(-\hat{E}_{2} \mid \mathcal{N}\right)<\infty$. For $\lambda \notin \Sigma^{*}, \lambda$ must be purely imaginary. Consider the invariant decomposition $\mathscr{N}=P_{\Sigma^{*}}(\mathscr{N}) \oplus\left(I-P_{\Sigma^{*}}\right)(\mathscr{N})=\left\{\left(v_{1}, v_{2}\right)\right\}$. For $\hat{E}_{2}\left(v_{2}\right) \geqq 0$, by Lemma 1 , (b), and the observation after that, index of $-\widehat{E}_{2} \mid P_{\Sigma^{*}}(\mathcal{N})=$ the index of $-\widehat{E}_{2} \mid \mathcal{N}$. By choosing a symplectic basis on the finite $2 \mathrm{~m}$-dimensional subspace $P_{\Sigma^{*}}(\mathscr{N})$, we have

$\dot{q}=R S q, \quad$ where $q \in R^{2 m}, \quad R=\left(\begin{array}{cc|ccc} & & -1 & \ddots & \\ & 0 & & & -1 \\ \hline 1 & \ddots & & 0\end{array}\right), \quad \hat{E}_{2}=\frac{1}{2}\langle q, S q\rangle$.

Let $\lambda_{1}, \ldots, \lambda_{2 m}$ be the eigenvalues of $R S$ and $s_{1}, \ldots s_{2 m}$ the eigenvalues of the symmetric matrix $S$.

$$
S_{1} \cdots S_{2 m}=\operatorname{det} S=\operatorname{det} R \operatorname{det} S=\operatorname{det} R S=\lambda_{1} \cdots \lambda_{2 m} \neq 0 \quad(\operatorname{det} R=1) .
$$

For eigenvalues of $R S$ occur in quadruple $\{\lambda,-\lambda, \bar{\lambda},-\bar{\lambda}\}$, the oddness of the index of $-\hat{E}_{2} \mid P_{\Sigma^{*}}(\mathcal{N})$, implies that $s_{1} \cdots s_{2 m}$ has a negative sign. Therefore, there must be odd number of pairs of eigenvalues in the form $\pm \lambda, \lambda>0$. This completes the proof of Theorem 4.

\section{Kirchhoff Elliptic Vortex}

Our basic example in which our basic theorems in Sects 2 and 3 can be applied is that of the Kirchhoff elliptic vortex $\chi_{K}$, where $K=\left\{(x, y) \mid\left(x^{2} / a^{2}\right)+\left(y^{2} / b^{2}\right) \leqq 1\right\}(a>b)$. This vortex $\chi_{K}$ rotates with angular velocity $\Omega=\left(a b /(a+b)^{2}\right)\left(<\frac{1}{4}\right)$. In this section, we only outline the processes and state the results. For detailed computations consult [26]. Let us introduce elliptic coordinates $(\xi, \eta)$ via $x+y i=c \cosh (\xi+i \eta)$, $c=\sqrt{a^{2}-b^{2}}$. The ellipse $\partial K$ is described by $\xi=\xi_{0}$ a constant function, with $c \cosh \xi_{0}=a, c \sinh \xi_{0}=b$.

By matching solutions of $-\Delta \psi=\chi_{K}$ inside and outside of $K$ along $\partial K$ as a $C^{1}$ function, we have

$$
\hat{\psi}=\left\{\begin{array}{l}
-\frac{a^{2} b^{2}}{2\left(a^{2}+b^{2}\right)}\left(\frac{x^{2}}{a^{2}}+\frac{y^{2}}{b^{2}}\right)+\hat{\psi}(0), \quad \text { inside } K, \\
-\frac{a b}{2} \xi-\frac{a b}{4} e^{-2 \xi} \cos 2 \eta+\frac{\Omega}{4} c^{2}\left[\cos ^{2} h \xi+\sin ^{2} h \xi+\cos 2 \eta\right]+\hat{\psi}(0)
\end{array}\right.
$$

outside $K$.

Choose a cross-section $\pi_{\alpha}$ by taking $\alpha=x y$, so $\mathcal{N}=$ orthogonal complement of $\cos \eta, \sin \eta, \cos 2 \eta, \sin 2 \eta$. It is not difficult to verify for $m=1,2, \ldots$ 
(i) $\int_{0}^{2 \pi} G\left(\xi_{0}+i \eta, \xi_{0}+i \eta^{\prime}\right) \cos m \eta d \eta=\frac{\cosh m \xi_{0}}{m e^{m \xi_{0}}} \cos m \eta^{\prime}$
(ii) $\int_{0}^{2 \pi} G\left(\xi_{0}+i \eta, \xi_{0}+i \eta^{\prime}\right) \sin m \eta d \eta=\frac{\sinh m \xi_{0}}{m e^{m \xi_{0}}} \sin m \eta^{\prime}$

Hence, the linear operator $\mathscr{L} q=-\left(a b /(a+b)^{2}\right) q+\int G q$ has eigenfunctions $\left\{\begin{array}{l}\cos m \eta \\ \sin m \eta\end{array}\right.$ with eigenvalues $\left\{\begin{array}{l}-\frac{a b}{(a+b)^{2}}+\frac{\cosh m \xi_{0}}{m e^{m \xi_{0}}} \\ -\frac{a b}{(a+b)^{2}}+\frac{\sinh m \xi_{0}}{m e^{m \xi_{0}}}\end{array}, \quad m=1,2, \ldots\right.$.

On $\mathscr{N}$, the largest eigenvalue of $\mathscr{L} q$ is given by $-\left(a b /(a+b)^{2}\right)+\left(\cosh 3 \xi_{0} /\right.$ $\left.3 e^{3 \xi_{0}}\right)=\left(a^{2}(a-3 b) / 3(a+b)^{3}\right)$. Therefore, $\hat{E}_{2}=\frac{1}{2}\langle q, \mathscr{L} q\rangle$ is negative definite if and only if $a<3 b$. One can verify that $\hat{\psi}^{-1}(\hat{\psi} \mid \partial A) \backslash \partial A$ is a simple closed curve, and it can enclose a disk $D$ (centered at the origin) which contains properly the ellipse $K$. Now, we can apply Theorems 1,2 and 4 to Kirchhoff vortex $\chi_{K}$ and conclude:

Theorem 5. (a) When $a<3 b, E$ has a non-degenerate local maximum at $\chi_{K}$ on $M_{0} \cap \pi_{\alpha} \cap\left\{\chi_{B} \mid B \subseteq D\right\}$. (b) When $a<3 b, \chi_{K}$ is $L^{1}$-stable relative to $D$. (c) When $a / b$ is slightly larger than $3,-\hat{E}_{2} \mid \mathscr{N}$ has index 1 . Hence, $\chi_{K}$ is not neutrally stable.

Write $q=\sum_{m \geqq 1}\left(A_{m} \cos m \eta+B_{m} \sin m \eta\right)$, the linearized equation

$$
\dot{q}=(\mathscr{L} q)_{\eta} \text { becomes: }\left\{\begin{array}{l}
\dot{A}_{m}=\left[-\frac{a b}{(a+b)^{2}} m+\frac{\sinh m \xi_{0}}{e^{m \xi_{0}}}\right] B_{m} \\
\dot{B}_{m}=\left[\frac{a b}{(a+b)^{2}} m-\frac{\cosh m \xi_{0}}{e^{m \xi_{0}}}\right] A_{m},
\end{array} \quad m=1,2, \ldots,\right.
$$

which is the same as that in Love [18]. On the invariant subspace [cos $m \eta, \sin m \eta]$, $(\mathscr{L} q)_{\eta}$ has eigenvalues $\pm i \lambda_{m}, \lambda_{m}^{2}=\frac{1}{4}\left[\left(\left(2 a b /(a+b)^{2}\right) m-1\right)^{2}-((a-b) /(a+b))^{2 m}\right]$. For $\lambda_{3}^{2}=\left(a^{2} b^{2} /(a+b)^{3}\right)(3 a-b)(3 b-a)$, we obtain that $\chi_{K}$ is not neutrally stable if $a>3 b$.

Since our energy function $E$, and the constraints both are invariant under a $Z_{2}$ action (rotation on $R^{2}$ by $180^{\circ}$ ). One can expect a pitchfork bifurcation at $a / b=3$. As suggested by Appendix (B), computations on $C^{1}(\eta)$ can be carried out in smooth setting.

Let us express a typical element in $\mathscr{L}^{2}(\eta)$ as $X+Y+Z$ with $X=x \cos 3 \eta$, $Y=\left(a_{0} / 2\right)+a_{1} \cos \eta+a_{2} \sin \eta+a_{3} \cos 2 \eta+a_{4} \sin 2 \eta,\left(x, a_{0}, \ldots, a_{4} \in R\right)$

$Z \in[\overline{\sin 3 \eta, \cos 4 \eta, \sin 4 \eta, \ldots]}$ (the closure of the linear span of $\sin 3 \eta, \cos 4 \eta, \ldots)$.

Expand $\hat{E}=\frac{1}{2}\langle\omega, \hat{\psi}\rangle$ in $x, Y, Z$ with $X+Y+Z \in C^{1}(\eta)$ :

$$
\hat{E}(x, Y, Z)=\frac{1}{2}\langle Z, \mathscr{L} Z\rangle+x^{2}\langle\boldsymbol{\alpha}, Z\rangle+x^{2}\langle\boldsymbol{\beta}, Y\rangle+\cdots+\gamma x^{4}+\cdots, \boldsymbol{\alpha}, \boldsymbol{\beta} \in C^{1}, \gamma \in R .
$$

Constraints are given as:

$$
a_{i}=s_{i} x^{2}+\left\langle t_{i}, Z\right\rangle+\cdots, s_{i} \in R, t_{i} \in C^{1}, i=0, \ldots, 4 .
$$


Restricting $\hat{E}$ to the constraints:

$$
\begin{aligned}
\hat{E}(x, Z) & =\frac{1}{2}\langle Z, \mathscr{L} Z\rangle+x^{2}\langle\hat{\alpha}, Z\rangle+\cdots+\hat{\gamma} x^{4}+\cdots \text { for some } \hat{\alpha} \in C^{1}, \hat{\gamma} \in R . \\
\frac{\partial \hat{E}}{\partial Z} & =Z+x^{2} \hat{\alpha}+\cdots=0 \text { implies } Z=-x^{2} \mathscr{L}^{-1} \hat{\alpha}+\cdots .
\end{aligned}
$$

Restricting $\hat{E}$ further to $(\partial \hat{E} / \partial Z)=0$ :

$$
\begin{aligned}
\hat{E}(x) & =\frac{1}{2}\left\langle-x^{2} \mathscr{L}^{-1} \hat{\alpha},-x^{2} \hat{\alpha}\right\rangle+x^{2}\left\langle\hat{\alpha},-x^{2} \mathscr{L}^{-1} \hat{\alpha}\right\rangle+\hat{\gamma} x^{4}+\cdots \\
& =\left(\hat{\gamma}-\frac{1}{2}\left\langle\alpha, \mathscr{L}^{-1} \hat{\alpha}\right\rangle\right) x^{4}+\cdots .
\end{aligned}
$$

The computations of $\boldsymbol{\alpha}$ (or $\boldsymbol{\beta}$ ) involve about 50 terms of integrations, some of them are not easy. The $\gamma$ involves about 30 terms. The complexity in computations discourages the author to find out the numerical value of the number $\hat{\gamma}-\frac{1}{2}\left\langle\hat{\alpha}, \mathscr{L}^{-1} \hat{\alpha}\right\rangle$. From the numerical results in [11], it suggests that $\hat{c}=$ $\hat{\gamma}-\frac{1}{2}\left\langle\hat{\alpha}, \mathscr{L}^{-1} \hat{\alpha}\right\rangle$ may be positive. If $\hat{c}>0$, then for $a / b$ slightly larger than 3 , there exists a stable elliptic-like rotating vortex $\chi_{B}$ having the same circulation, centre, and angular momentum as that of the unstable elliptic vortex $\chi_{K}$, $K=\left\{(x, y) \mid\left(x^{2} / a^{2}\right)+\left(y^{2} / b^{2}\right) \leqq 1\right\}$.

\section{Finite Kelvin Waves $\mathscr{K}_{m}$}

Consider wave motions of the boundary of a patch $\chi_{B}$ near a circular patch $\chi_{U}$, with $U$ the unit disk. Thus, we examine the linearized equation of motion in a rotating frame with a suitable angular velocity $\Omega$. Let $(r, \theta)$ be the polar coordinates fixed in the rotating frame.

Following the approach in Sect. 3, $\hat{\psi}=\psi+\frac{1}{2} \Omega r^{2}$,

$$
\begin{aligned}
\psi & =\left\{\begin{array}{ll}
\frac{1}{4}\left(1-r^{2}\right)+c, & r \leqq 1, \\
\frac{1}{2} \ln \frac{1}{r}+c, & r \geqq 1,
\end{array} \text { for some constant } c\left(=\psi(0)-\frac{1}{4}\right) .\right. \\
\mathscr{L} q & =\hat{\psi}_{\xi} q+\int G q=\left(-\frac{1}{2}+\Omega\right) q+\int G q . \text { Thus, } \\
\mathscr{L}(\cos m \eta) & =\left(-\frac{1}{2}+\Omega+\frac{1}{2 m}\right) \cos m \eta, \\
\mathscr{L}(\sin m \eta) & =\left(-\frac{1}{2}+\Omega+\frac{1}{2 m}\right) \sin m \eta, \quad m \geqq 1 .
\end{aligned}
$$

On the subspace spanned by $\cos m \eta, \sin m \eta, q=A_{m} \cos m \eta+B_{m} \sin m \eta$, one has

$$
\left\{\begin{array}{l}
\dot{A}_{m}=\left[\left(-\frac{1}{2}+\Omega\right) m+\frac{1}{2}\right] B_{m}, \\
\dot{B}_{m}=-\left[\left(-\frac{1}{2}+\Omega\right) m+\frac{1}{2}\right] A_{m} .
\end{array}\right.
$$

Hence, for $\Omega=\Omega_{m}=(m-1) / 2 m, \dot{q}=0$ one finds an infinitesimal wave rotating with angular velocity $\Omega$ in the shape $r=1+A_{m} \cos m \eta+B_{m} \sin m \eta\left(A_{m}, B_{m}\right.$ small). These waves are called the (infinitesimal) Kelvin waves.

Kirchhoff vortex can be regarded as a finite version of Kelvin waves with $m=2$. 
Recently, finite version of Kelvin waves $\mathscr{K}_{m}$ with $m>2$ has been obtained both numerically and theoretically $[7,8]$. According to Burbea, $\mathscr{K}_{m}$ can be represented in complex coordinates as $\chi_{\phi(\mid \omega) \leqq 1)}$; where

$$
\phi(\omega)=\omega\left[1+\varepsilon \omega^{-m}+\beta_{2} \omega^{-2 m}+\cdots\right],
$$

with $\beta_{n}=0\left(\varepsilon^{2}\right)$ for $n \geqq 2$

$$
\Omega=\Omega_{m}^{\varepsilon}=\frac{1}{2} \frac{m-1}{m}\left\{1-\frac{m}{2} \varepsilon^{2}+\cdots\right\}, \quad m=2,3, \ldots
$$

for $\varepsilon>0$ and small.

These solutions are invariant under $Z_{m}$-action generated by rotation $z \rightarrow z e^{2 \pi i / m}$.

Let us examine the energy function $E$ near $\mathscr{K}_{m}$ for a fixed $m, m>3$, through the rest of this section. We choose $\alpha=\operatorname{Im} z^{m}$ (the imaginary part of $z^{m}$ ) to define the cross-section. Consider $\mathscr{L}^{\varepsilon} q=r^{\varepsilon} q+\int G^{\varepsilon} q$ with $r^{\varepsilon}=(\partial \hat{\psi} / \partial \xi) / I_{0}$. In the limit as $\varepsilon \searrow 0$, $\mathscr{N}^{0}=\left\{q \in Q \mid \int(\cos \eta) q=\int(\sin \eta) q=\int(\cos m \eta) q=\int(\sin m \eta) q=0\right\}$, and $\mathscr{L}^{0}\left\{\begin{array}{l}\cos m \eta \\ \sin m \eta\end{array}\right\}$ $=\left(-\frac{1}{2}+\Omega+\frac{1}{2 m}\right)\left\{\begin{array}{l}\cos m \eta \\ \sin m \eta\end{array}\right\}$. Thus, $-\hat{E}_{2}=-\frac{1}{2}\left\langle q, \mathscr{L}^{0} q\right\rangle$ on $\mathscr{N}^{0}$ is nondegenerate and of even index $2(m-2)$. So does $-\widehat{E}_{2}$ on $\mathcal{N}^{\varepsilon}$ for small $\varepsilon>0$. Therefore, $\hat{E}$ is neither a local maximum nor a local minimum on the $M_{0} \cap \pi_{\alpha}$, for small $\varepsilon>0$. To obtain a variational characterization, we need to restrict ourselves further to $Z_{m}$-symmetric perturbations.

Let $\mathscr{N}_{m}^{0}, \mathscr{N}_{m}^{\varepsilon}, \mathscr{V}_{m}$ be subspaces of fixed vectors in $\mathscr{N}^{0}$ and $\mathscr{N}^{\varepsilon}, \mathscr{V}$ under the $Z_{m^{-}}$ action respectively. For $\hat{E}_{2}$ is negative definite on $\mathscr{N}_{m}^{0}, \hat{E}_{2}$ is negative definite on $\mathscr{N}_{m}^{\varepsilon}$ for $\varepsilon>0$ small. Let $d_{m}^{*}>1$ be the unique solution of the equation $\hat{\psi}(1)=\hat{\psi}(d)$ or lnd $=\Omega_{m}^{\varepsilon}\left(d^{2}-1\right)$. Denote by $D$ a disk centered at the origin with radius $d_{m}$, $1<d_{m}<d_{m}^{*}$. By suitable modifications of the proofs of Theorems 1 and 2, we obtain:

Theorem 7. (a) For $\varepsilon>0$ and small, the energy E has a non-degenerate local maximum at $\mathscr{K}_{m}$ on $\left\{\chi_{B} \in M_{0} \cap \pi_{\alpha} \mid \chi_{B} \in \mathscr{V}_{m}, B \subseteq D\right\}$. (b) For $\varepsilon>0$ and small, $\mathscr{K}_{m}$ is $L^{1}$-stable in $\mathscr{V}_{m}$ relative to $D$. (Notice that $\mathscr{V}_{m}$ is invariant under the flow $\Phi_{t}$.)

Next we consider the question of linearized stability for $\mathscr{K}_{m}$. The above theorem only insures neutral stability under $Z_{m}$-symmetric perturbations. In the limit $\varepsilon \searrow 0$, $\left(\mathscr{L}^{0} \cos n \eta\right)_{\eta}=\left[\left(-\frac{1}{2}+\Omega_{m}\right) n+\frac{1}{2}\right] \sin \eta,\left(\mathscr{L}^{0} \sin \eta\right)_{\eta}=-\left[\left(-\frac{1}{2}+\Omega_{m}\right) n+\frac{1}{2}\right] \cos \eta$, $n \geqq 1$. So that the limit system has eigenvalues $\pm i\left[\left(-\frac{1}{2}+\Omega_{m}\right) n+\frac{1}{2}\right]$ and is neutrally stable. One expects that:

Theorem 8. For $\varepsilon$ small and positive, $\mathscr{K}_{m}$ is neutrally stable.

Proof. Consider the invariant decomposition $\mathscr{N}^{\varepsilon}=P_{\Gamma_{\varepsilon}}\left(\mathscr{N}^{\varepsilon}\right) \oplus\left(I-P_{\Gamma_{\varepsilon}}\right)\left(\mathscr{N}^{\varepsilon}\right)$ into symplectic subspaces. Here, $\Gamma_{\varepsilon}(\varepsilon \geqq 0)$ denotes the portion of eigenvalues branching off from $\pm i\left[\left(-\frac{1}{2}+\Omega_{m}\right) n+\frac{1}{2}\right]$ for $2 \leqq n<m$. At $\varepsilon=0, \hat{E}_{2}$ is negative definite on $\left(I-P_{\Gamma_{0}}\right)\left(\mathscr{N}^{0}\right)$ and positive definite on $P_{\Gamma_{0}}\left(\mathscr{N}^{0}\right)$. Thus, for $\varepsilon$ small and positive, $\hat{E}_{2}$ is again negative definite on $\left(I-P_{\Gamma_{\varepsilon}}\right)\left(\mathscr{N}^{\varepsilon}\right)$ and positive definite on $P_{\Gamma_{\varepsilon}}\left(\mathscr{N}^{\varepsilon}\right)$. Consequently, $(\mathscr{K} q)_{\eta}$ has only purely imaginary eigenvalues (near $\pm i\left[\left(-\frac{1}{2}+\right.\right.$ $\left.\left.\Omega_{m}\right) n+\frac{1}{2}\right]$ ), and $\mathscr{K}_{m}$ is neutrally stable. 
Remark 3. The Kelvin waves $\mathscr{K}_{m}$ is an example, where $-\hat{E}_{2} \mid \mathscr{N}$ has an even index and the system is neutrally stable.

Remark 4. As pointed out by a referee, the bifurcation theory of Kelvin waves should eventually be put into the context of Hamiltonian theory with $S^{1}$ symmetry that is currently under investigation by Golubisky, Stewart, and Marsden.

\section{A Co-rotating Pair}

We like to study a co-rotating pair $\chi_{W}, W=W_{0} \cup W_{1}$, as a desingularization of a pair of point vortices, $( \pm 1,0)$. Denote by $\left(r_{0}, \theta_{0}\right),\left(r_{1}, \theta_{1}\right)$ the polar coordinates centered at $(1,0),(-1,0)$ with polar axes in the directions $(1,0),(-1,0)$. Write $d a_{j}=r_{j} d r_{j} d \theta_{j}$, $j=0,1$, as the area elements.

Set $\mathfrak{U}=\left\{\left(\mathbf{r}_{0}, \mathbf{r}_{1}\right) \mid \mathbf{r}_{j} \in C^{1}\right.$ on $\left.\theta_{j}, 0<\mathbf{r}_{j}\left(\theta_{j}\right)<1, j=0,1\right\}$. To each $\left(\mathbf{r}_{0}, \mathbf{r}_{1}\right) \in \mathfrak{A}$, let $W_{j}=\left\{\boldsymbol{r}_{j} \leqq \mathbf{r}_{j}\left(\theta_{j}\right)\right\}, j=0,1, W=W_{0} \cup W_{1}$. Thus $\mathfrak{A}$ can be regarded as a subclass of vortex patches having radial boundaries via $\left(\mathbf{r}_{0}, \mathbf{r}_{1}\right) \rightarrow \chi_{W_{0} \cup W_{1}}$. We put a $C^{1}$-topology on $\mathfrak{U}$.

As suggested by Theorem 1, consider the following optimization problem. The solution will be a rotating vortex patch with a nice variational characterization. Let $C^{0}=\int_{W_{0}} d a_{0}, C^{1}=\int_{W_{1}} d a_{1}, S=\int_{W_{0}} y d a_{0}-\int_{W_{1}} y d a_{1}$ and recall $\bar{x}_{0}=\int x \omega, \bar{y}_{0}=\int y \omega$.

(CP) Maximize $E=\frac{1}{2}\langle\omega, \psi\rangle$ in $\mathfrak{A}$, subject to $C^{0}=C^{1}=$ constant, $\bar{x}_{0}=\bar{y}_{0}=0$, and $S=0$. The functions $E$ (by Proposition 3 in Appendix (B)), $C^{0}, C^{1}, J, \bar{x}_{0}, \bar{y}_{0}, S$ are all smooth on $\mathfrak{U}$ (with the $C^{1}$-topology). This is our technical reason for restricting our attention to the class $\mathfrak{U}$ for a moment, so that one can restore or carry out the analysis in a smooth setting. The condition $S=0$ is imposed so one can eliminate the $S^{1}$-symmetry of our problem.

Rescale the variables $\varepsilon R_{0}=r_{0}, \varepsilon R_{1}=r_{1}, \varepsilon>0$ one sees

$$
\begin{aligned}
J / \varepsilon^{2}= & \left(2 \varepsilon \int_{W_{0}} R_{0} \cos \theta_{0} d A_{0}+\varepsilon^{2} \int_{W_{0}} R_{0}^{2} d A_{0}\right)+\left(2 \varepsilon \int_{W_{1}} R_{1} \cos \theta_{1} d A_{1}+\varepsilon^{2} \int_{W_{1}} R_{1}^{2} d A_{1}\right) \\
& +\int_{W_{0}} d A_{0}+\int_{W_{1}} d A_{1} . \quad\left(\text { Here }, \varepsilon^{2} d A_{i}=d a_{i} .\right)
\end{aligned}
$$

And

$$
E / \varepsilon^{4}=\frac{1}{2}\left\langle W, \Psi^{\varepsilon}\right\rangle=\frac{1}{2}\left(\int_{W_{0}} \Psi^{\varepsilon} d A_{0}+\int_{W_{1}} \Psi^{\varepsilon} d A_{1}\right) \quad \text { with } \quad \Psi^{\varepsilon}=\psi / \varepsilon^{2}
$$

Take $\varepsilon \searrow 0$ by dropping some unimportant constant depending on $\varepsilon$. One is led to:

$\left(\mathrm{CP}^{0}\right)$ Maximize $E^{0}=\frac{1}{2}\left\langle W, \Psi^{0}\right\rangle$, subject to $\int_{W_{0}} d A_{0}=\int_{W_{1}} d A_{1}=$ constant,

$$
\begin{aligned}
& \int_{W_{0}} R_{0} \cos \theta_{0} d A_{0}+\int_{W_{1}} R_{1} \cos \theta_{1} d A_{1}=0, \\
& \int_{W_{0}} R_{0} \cos \theta_{0} d A_{0}-\int_{W_{1}} R_{1} \cos \theta_{1} d A_{1}=0, \\
& \int_{W_{0}} R_{0} \sin \theta_{0} d A_{0}-\int_{W_{1}} R_{1} \sin \theta_{1} d A_{1}=0, \\
& \int_{W_{0}} R_{0} \sin \theta_{0} d A_{0}+\int_{W_{1}} R_{1} \sin \theta_{1} d A_{1}=0,
\end{aligned}
$$


with

$$
\begin{aligned}
& \Psi^{0}\left(R_{0} e^{i \theta_{0}}\right)=\frac{1}{2 \pi} \int_{W_{0}} \ln \frac{1}{\left|R_{0} e^{i \theta_{0}}-R_{0}^{\prime} e^{i \theta_{0}^{\prime}}\right|} d A_{0}^{\prime}+c, \\
& \Psi^{0}\left(R_{1} e^{i \theta_{1}}\right)=\frac{1}{2 \pi} \int_{W_{1}} \ln \frac{1}{\mid R_{1} e^{i \theta_{1}}-R_{1}^{\prime} e^{i \theta_{1}^{\prime} \mid}} d A_{1}^{\prime}+c .
\end{aligned}
$$

(Here, $c$ is a suitable constant.)

Hence, the problem $\left(\mathrm{CP}^{0}\right)$ becomes decoupled. Namely, for $j=0,1$, we maximize $E_{j}^{0}=\frac{1}{2}\left\langle W_{j}, \Psi_{j}^{0}\right\rangle$, subject to $\int_{W_{j}} d A_{j}=$ constant, $\int R_{j} \cos \theta_{j} d A_{j}=\int R_{j} \sin \theta_{j} d A_{j}=0$, with $\Psi_{j}^{0}=(1 / 2 \pi) \int \ln \left(1 /\left|R_{j} e^{i \theta_{j}}-R_{j}^{\prime} e^{i \theta_{j}^{\prime}}\right|\right) d A_{j}^{\prime}+c$. Observe, $R_{j}=1$ (i.e. a circular vortex) is a solution of the above problem. Indeed, $E_{2}^{0}=\frac{1}{2}\left\langle q, \mathscr{L}^{0} q\right\rangle, q=\left(\mathbf{r}_{0}, \mathbf{r}_{1}\right)$, $\mathscr{L}^{0}=\mathscr{L}_{0}^{0} \oplus \mathscr{L}_{1}^{0}, \quad$ and $\quad \mathscr{L}_{j}^{0}\left\{\begin{array}{c}\cos m \eta_{j} \\ \sin m \eta_{j}\end{array}\right\}=\left(-\frac{1}{2}+(1 / 2 m)\right)\left\{\begin{array}{c}\cos m \eta_{j} \\ \sin m \eta_{j}\end{array}\right\}, \quad j=0,1, m \geqq 2$. Thus, $E_{2}^{0}$ is negative definite on $\mathscr{N}^{0}$. By continuity, this problem has a solution for small $\varepsilon>0$.

Theorem 9. There exists a $Z_{2}$-symmetric co-rotating vortex pair $\mathscr{T}_{2}$, for $\varepsilon>0$ small, in the form $r_{j}=\varepsilon+o(\varepsilon), j=0,1$. Furthermore, they are $L^{1}$-stable in any fixed large disk $D$ centered at the origin.

Proof. We use Theorem 2 to get the second part of this theorem. Now it is only necessary to check the condition (2) in that theorem. Computations show $\left.\psi\right|_{\partial W}=$ $0\left(\varepsilon^{2}\right),\left.\quad \psi\right|_{\partial D} \leqq-\varepsilon^{2}(\ln (1 / \varepsilon)+0(1))$, and $\Omega=0\left(\varepsilon^{2}\right)$. Thus $\tilde{\psi}|\partial W-\tilde{\psi}| \partial D \geqq-\varepsilon^{2}$ $(\ln (1 / \varepsilon)+0(1))>0$, as $\varepsilon>0$ and small, and the condition (2) in Theorem 2 is verified.

Our solution can be continued and when $\varepsilon$ get large, the vortex pair will lose its neutral stability. Numerical computations are found in $[25,11]$. It seems that 0 remains to be a double eigenvalue before the vortex pair loses its neutral stability at $\varepsilon=\varepsilon_{1}$. Thus, $\hat{E}_{2} \mid \mathcal{N}$ is non-degenerate for all $\varepsilon, 0 \leqq \varepsilon<\varepsilon_{1}$. For $\widehat{E}_{2} \mid \mathcal{N}$ is negative definite at $\varepsilon=0, \hat{E}_{2} \mid \mathcal{N}$ remains to be negative definite for all $\varepsilon, 0<\varepsilon<\varepsilon_{1}$. Consequently, we obtain a nonlinear stability in a $L^{1}$-sense, for any $\varepsilon, 0<\varepsilon<\varepsilon_{1}$.

\section{Co-Rotating $m$ Uniform Vortices $(m \geqq 3$ )}

Finally, let us examine co-rotating $m$ uniform vortices as a desingularization of Thomson's $m$ point vortices. There is a natural action of $Z_{m}$ on $R^{2}$, generated by rotating through angle $2 \pi / m$. We find it is convenient to use complex notation in the plane: $z=x+y i$. Let $\tau=e^{i 2 \pi / m}$. Thomson's $m$ point vortices consists of $m$ points $1, \tau, \tau^{2}, \ldots, \tau^{m-1}$ on the unit circle which are invariant under the natural $Z_{m}$-action on $R^{2}(\approx \mathbb{C})$. Denote by $\left(r_{j}, \theta_{j}\right)$, the polar coordinates around each $\tau^{j}$, i.e. $z_{j}=$ $\tau^{j}\left(1+r_{j} e^{i \theta_{j}}\right)$, for $j=0,1, \ldots, m-1$.

As suggested by Theorem 1, let us examine an Optimization problem for $a Z_{m^{-}}$ invariant vortex patch under $Z_{m}$-invariant perturbations. Set $\mathfrak{U}=\left\{\mathbf{r} \in C^{1}(\theta) \mid 0<\mathbf{r}(\theta)\right.$ $<\sin (\pi / m)\}$. To each $\mathbf{r} \in \mathfrak{A}$. $W_{j}=W_{j}(\mathbf{r})=\left\{z_{j}=\tau^{j}\left(1+r_{j} e^{i \theta_{j}}\right) \mid r \leqq \mathbf{r}(\theta)\right\}$, and $W=W(\mathbf{r})$ $=W_{0} \cup \cdots \cup W_{m-1}$. Thus $\chi_{W_{0}}$ is the portion of $\chi_{W}$ in the sector $\{z||$ argument $z \mid$ $<(\pi / m)\}$. (CN) Maximize $E\left(\chi_{W}\right)$ on $\mathfrak{U}$ subject to $\int_{W_{0}} d a=$ constant, $J\left(\chi_{W_{0}}\right)=$ const- 
ant, $S\left(\chi_{W_{0}}\right)=\int_{W_{0}} y d a=0 .\left(d a=r_{0} d r_{0} d \theta_{0}\right)$. The condition $S=0$ is used to eliminate the $S^{1}$-symmetry of this problem.

Rescale the variables $\varepsilon R=r, \varepsilon>0$. Notice that,

$$
\begin{aligned}
& j / \varepsilon^{2}=2 \varepsilon \int_{W_{0}} R \cos \theta d A+\varepsilon^{2} \int_{W_{0}} R^{2} d A+\int_{W_{0}} d A\left(\varepsilon^{2} d A=d a\right), \\
& E / \varepsilon^{4}=\frac{1}{2}\left\langle W, \Psi^{\varepsilon}\right\rangle=\frac{m}{2}\left\langle W_{0}, \Psi^{\varepsilon}\right\rangle,
\end{aligned}
$$

and

$$
\begin{aligned}
\Psi^{\varepsilon}\left(R e^{i \theta}\right)= & \psi / \varepsilon^{2}=\frac{1}{2 \pi} \int_{W_{0}} \ln \frac{1}{\mid \varepsilon R e^{i \theta}-\varepsilon R^{\prime} e^{i \theta^{\prime} \mid}} R^{\prime} d R^{\prime} d \theta^{\prime} \\
& +\sum_{j=1}^{m-1} \frac{1}{2 \pi} \int_{W_{0}} \frac{1}{\left|\left(1+\varepsilon R e^{i \theta}\right)-\tau^{j}\left(1+\varepsilon R^{\prime} e^{i \theta^{\prime}}\right)\right|} R^{\prime} d R^{\prime} d \theta^{\prime} .
\end{aligned}
$$

Let $\varepsilon \searrow 0$ and consider the limit problem, we have $\left(\mathrm{CN}^{0}\right)$ Maximize $E^{0} / \mathrm{m}=$ $\frac{1}{2}\left\langle W_{0}, \Psi^{0}\right\rangle$ on $\mathfrak{A}$ subject to $\int_{W_{0}} d A=$ constant,

$$
\begin{aligned}
\int_{W_{0}} R \cos \theta d A & =\int_{W_{0}} R \sin \theta d A=0, \text { with } \\
\Psi^{0} & =\frac{1}{2 \pi} \int_{W_{0}} \ln \frac{1}{\left|R e^{i \theta}-R^{\prime} e^{i \theta^{\prime}}\right|} R^{\prime} d R^{\prime} d \theta^{\prime}+\text { constant. }
\end{aligned}
$$

Observe that $R=1$ (i.e. a circular vortex) is a solution of this problem $\left(\mathrm{CN}^{0}\right)$. Indeed, $E_{2}^{0}$ is negative definite on the subspace $\mathscr{N}_{m}^{0}=\left\{\left(k_{1}, \ldots, k_{m}\right) \in \mathscr{N}^{0} \mid k_{1}=\cdots\right.$ $\left.=k_{m}\right\}$, which is fixed by the $Z_{m}$-action on $\mathscr{N}^{0}$. Therefore, $E_{2}^{\varepsilon}$ is negative definite on the subspace $\mathscr{N}_{m}^{\varepsilon}=\left\{\left(k_{1}, \ldots, k_{m}\right) \in \mathscr{N}^{\varepsilon}\right\}$ for $\varepsilon>0$ and small. As before (cf. Theorems 9,7), we have

Theorem 10. There exists a $Z_{m}$-symmetric co-rotating patch $\mathscr{T}_{m}$ for $\varepsilon>0$ and small in the form $r_{j}=\varepsilon+0(\varepsilon), j=0,1, \ldots, m-1$. Furthermore, they are $L^{1}$-stable under $Z_{m^{-}}$ symmetric perturbations in any fixed large disk $D$ centered at the origin.

Now let us determine the indices and the neutral stability for these vortices $\mathscr{T}_{m}$ obtained in Theorem 9 above. The results will be presented as Theorem 10, 11 and 12. With our normalization (slightly different from that in [9]), we can readily establish, $r_{j}=\varepsilon+\varepsilon^{3} c_{m} \cos 2 \theta_{j}+0\left(\varepsilon^{4}\right), \quad \Omega=(m-1 / 4) \varepsilon^{2}\left(1+0\left(\varepsilon^{2}\right)\right)$, with $c_{m}=-(m$ -1) $(m-5) / 12$ (need Lemma 9). Write $\mathscr{L}^{\varepsilon}=\mathscr{L}_{0}+\varepsilon \mathscr{L}_{1}+\varepsilon^{2} \mathscr{L}_{2}+0\left(\varepsilon^{3}\right)$ on $Q$, long computations show

$$
\begin{aligned}
\mathscr{L}_{0}\left(\left(k_{j}\right)\right) & =\left(-\frac{1}{2} k_{j}+\frac{1}{2 \pi} \int \ln \frac{1}{\mid e^{i \theta_{j}}-e^{i \theta_{j} \mid}} k\left(\theta_{j}^{\prime}\right) d \theta_{j}^{\prime}\right), \\
\mathscr{L}_{1} & =\left(-\frac{1}{2 \pi} \Sigma_{l}^{\prime} \operatorname{Re} \frac{\tau^{j} e^{i \theta_{j}}-\tau^{l} e^{i \theta_{l}}}{\tau^{j}-\tau^{l}} k_{l}\left(\theta_{l}\right) d \theta_{l}\right),
\end{aligned}
$$


and

$$
\left(\begin{array}{rl}
\mathscr{L}_{2}= & \frac{1}{2}\left[\Sigma_{l}^{\prime} \operatorname{Re}\left(\frac{\tau^{j} e^{i \theta_{j}}}{\tau^{j}-\tau^{l}}\right)^{2}\right] k_{j}+\frac{m-1}{4} k_{j} \\
& +\frac{1}{4 \pi} \Sigma_{l}^{\prime} \int\left(\frac{\tau^{j} e^{i \theta_{j}}-\tau^{l} e^{i \theta_{l}}}{\tau^{j}-\tau^{l}}\right)^{2} k_{l}\left(\theta_{l}^{\prime}\right) \mathrm{d} \theta_{l}^{\prime} \\
& -\frac{c_{m}}{2 \pi} \int \operatorname{Re}\left(\frac{\cos 2 \theta_{j} e^{i \theta_{j}}-\cos 2 \theta_{j}^{\prime} e^{i \theta_{j}^{\prime}}}{e^{i \theta_{j}}-e^{i \theta_{j}}}\right) k\left(\theta_{j}^{\prime}\right) d \theta_{j}^{\prime}
\end{array}\right) .
$$

Here, $\Sigma_{l}^{\prime}$ means the summation without the term with index $l=j$.

For small $\varepsilon \geqq 0$, decompose $Q=V^{\varepsilon} \oplus Y^{\varepsilon}$ according to the separation of the spectrum of $\left(\mathscr{L}^{\varepsilon} q\right)_{\eta}$ into parts near the origin and the parts away from the origin. Thus $V^{0}=\underset{j}{X}\left[\cos \theta_{j}, \sin \theta_{j}\right], Y^{0}=\underset{j}{X}\left[\cos \theta_{j}, \sin \theta_{j}\right]^{\perp}$. Let $Z^{\varepsilon}=\left[x_{\eta}, y_{\eta}, \alpha_{\eta},\left(x^{2}+y^{2}\right)_{\eta}\right]$, with a cross-section defined through $\alpha=\operatorname{Im}\left(\tau^{-j} z_{j}^{m}\right)$ near $\tau^{j}, j=0,1, \ldots, m-1$. For $\Omega=$ $O\left(\varepsilon^{2}\right), Z^{\varepsilon} \subset V^{\varepsilon}$ and $N^{\varepsilon} \supset Y^{\varepsilon}$. Let $X^{\varepsilon}=\mathscr{N}^{\varepsilon} \cap V^{\varepsilon}$ so $X^{\varepsilon}=$ skew-orthogonal subspace of $Z^{\varepsilon}$ in $V^{\varepsilon}$. Hence, we have a decomposition of $Q$ into symplectic subspaces: $Q=X^{\varepsilon} \oplus Y^{\varepsilon} \oplus Z^{\varepsilon}=\left\{\left(x^{\varepsilon}, y^{\varepsilon}, z^{\varepsilon}\right)\right\}(\varepsilon \geqq 0)$.

Elements of $Y^{\varepsilon}$ can be represented as $y^{\varepsilon}=y^{0}+\varepsilon x^{0}\left(y^{0}\right)+\varepsilon z^{0}\left(y^{0}\right)+0\left(\varepsilon^{2}\left|y^{0}\right|\right)$ for some linear functionals $x^{0}=x^{0}\left(y^{0}\right), z^{0}=z^{0}\left(y^{0}\right)$ on $Y^{0}$. Thus,

\section{Lemma 2.}

$$
\begin{aligned}
\frac{1}{2}\left\langle y^{\varepsilon}, \mathscr{L}^{\varepsilon} y^{\varepsilon}\right\rangle & =\frac{1}{2}\left\langle y^{0}+\varepsilon x^{0}+\varepsilon z^{0}+\cdots,\left(\mathscr{L}_{0}+\varepsilon \mathscr{L}_{1}+\cdots\right)\left(y^{0}+\varepsilon x^{0}+\varepsilon z^{0}+\cdots\right)\right\rangle \\
& =\frac{1}{2}\left\langle y^{0}, \mathscr{L}_{0} y^{0}\right\rangle+0\left(\varepsilon\left|y^{0}\right|^{2}\right)<0 .
\end{aligned}
$$

Elements of $X^{\varepsilon}$ can be represented as $x^{\varepsilon}=x^{0}+\varepsilon^{2} y^{0}\left(x^{0}\right)+\varepsilon z^{0}\left(x^{0}\right)+\varepsilon^{2} z^{1}\left(x^{0}\right)+$ $O\left(\varepsilon^{3}\left|x^{0}\right|\right)$. Notice that $\operatorname{ker} \mathscr{L}_{0}=V^{0}$, Image $\mathscr{L}_{0}=Y^{0}$, and $\mathscr{L}_{1}\left(V^{0}\right)=$ const. Thus,

$$
\begin{aligned}
\frac{1}{2}\left\langle x^{\varepsilon}, \mathscr{L}^{\varepsilon} x^{\varepsilon}\right\rangle= & \frac{1}{2}\left\langle x^{0}+\varepsilon^{2} y^{0}+\varepsilon z^{0}+\cdots,\left(\mathscr{L}_{0}+\varepsilon \mathscr{L}_{1}+\varepsilon^{2} \mathscr{L}_{2}+\cdots\right)\right. \\
& \left.\cdot\left(x^{0}+\varepsilon^{2} y^{0}+\varepsilon z^{0}+\cdots\right)\right\rangle \\
= & \frac{\varepsilon^{2}}{2}\left\langle x^{0}, \mathscr{L}_{2} x^{0}\right\rangle+0\left(\varepsilon^{3}\left|x^{0}\right|^{2}\right) .
\end{aligned}
$$

Using the transformation from $\left(k_{j}\right) \in V^{0}$ onto $\zeta=\left(\zeta_{j}\right) \in \mathbb{C}^{n}$ via $\left(k_{j}\right)=\varepsilon\left(\operatorname{Re} \zeta_{j} \cos \theta_{j}\right.$ $+\operatorname{Im} \zeta_{j} \sin \theta_{j}$ ), the subspace $X^{0}$ goes to

$$
[e, i e]^{\perp} \oplus[i \operatorname{Re} \gamma+\operatorname{Im} \gamma,-\operatorname{Re} \gamma+i \operatorname{Im} \gamma]^{\perp}\left(e=(1, \ldots, 1), \gamma=\left(1, \tau, \ldots, \tau^{m-1}\right)\right) .
$$

One can readily verify by brute force computations that $\left(\varepsilon^{2} / 2\right)\left\langle x^{0}, \mathscr{L}_{2} x^{0}\right\rangle=$ $\left(\pi \varepsilon^{4} / 2\right)\langle\zeta, L \zeta\rangle$, with $L$ defined in Appendix (C) before Lemma 11. Thus,

Lemma 3. $\frac{1}{2}\left\langle x^{\varepsilon}, \mathscr{L}^{\varepsilon} x^{\varepsilon}\right\rangle=\left(\pi \varepsilon^{4} / 2\right)\left(\langle\zeta, L \zeta\rangle+0\left(\varepsilon|\zeta|^{2}\right)\right)$.

For $m \leqq 6,\langle\zeta, L \zeta\rangle$ is positive definite by Proposition 4(a) in the Appendix (C). Lemmas 1, 2, 3 and the above fact imply the following result.

Theorem 11. Let $m \leqq 6$. For $\varepsilon>0$ and small, we have

(1) $-\hat{E}_{2} \mid \mathscr{N}^{\varepsilon}$ is of index $2 m-4$,

(2) the system is neutrally stable. 
For $m \geqq 8$, the story about $\langle\zeta, L \zeta\rangle$ becomes complicated. Lemmas $1,2,3$ Propositions 4 (c) and 5 in the Appendix 3 provide us the following information about $\hat{E}$.

Theorem 12. Let $m \geqq 8$. For $\varepsilon>0$ and small, we get

(1) $-\hat{E}_{2} \mid \mathscr{N}^{\varepsilon}$ is of saddle type with index $\leqq 2 m-4$.

(2) $-\hat{E}_{2} \mid \mathscr{N}^{\varepsilon}$ is of odd index iff the number $m$ is even.

By Theorem 4 in Sect. 3, the system is unstable for $m \geqq 8$ and $m$ even. Our detailed computations of the linearized equation enable us to examine the question of neutral stability for any $m, m \neq 7$.

Indeed, we obtain in addition that:

Theorem 13. For $\varepsilon>0$ and small, the $Z_{m}$-symmetric uniform $m$ vortices $\mathscr{T}_{m}$ is not neutrally stable for $m \geqq 8$.

Proof. The induced symplectic structure on $\mathbb{C}^{m}$ via $x^{\varepsilon} \rightarrow \zeta\left(x^{0}=\varepsilon\left(\operatorname{Re} \zeta_{j} \cos \theta_{j}\right.\right.$ $\left.\left.+\operatorname{Im} \zeta_{j} \sin \theta_{j}\right)\right)$ is given by $\left(\zeta, \zeta^{\prime}\right) \rightarrow \pi \varepsilon^{2}\left\langle i \zeta, \zeta^{\prime}\right\rangle$. Recall, $\frac{1}{2}\left\langle x^{\varepsilon}, \mathscr{L}^{\varepsilon} x^{\varepsilon}\right\rangle=\left(\pi \varepsilon^{4} / 2\right)\langle\zeta, L \zeta\rangle$ $+0\left(\varepsilon^{5}|\zeta|^{2}\right)$. Therefore, the linear Hamiltonian system $\dot{q}=\left(\mathscr{L}^{\varepsilon} q\right)_{\eta}$ on $X^{\varepsilon}$ can be expressed as $\dot{\zeta}=-i \varepsilon^{2}(L \zeta+O(\varepsilon|\zeta|))$, which is a "small" perturbation of the linear Hamiltonian system $\dot{\zeta}=-i \varepsilon^{2} L \zeta$ in the Appendix (C). By Proposition 6 in Appendix (C), $-\varepsilon^{2}(L \zeta+O(\varepsilon|\zeta|))$ has a pair of real eigenvalues $\pm \varepsilon^{2}[\lambda+O(\varepsilon)], \lambda>0$. Therefore, the system $\dot{q}=\left(\mathscr{L}^{\varepsilon} q\right)_{\eta}$ is not neutrally stable.

Remark 5. The vortex patch $\mathscr{T}_{2 s+1}, s \geqq 4$ is an example with $-\hat{E}_{2}$ of even index and yet is not stable.

\section{Appendices}

\section{(A) Discreteness of the Spectra of $(\mathscr{L} q)_{\eta}$ and $(\mathscr{K} q)_{\eta}$.}

As before, let $(\xi, \eta)$ be a smooth coordinate system $x=x(\xi, \eta), y=y(\xi, \eta)$ near a $C^{1}$ boundary of a vortex patch $\chi_{A}$ so that $\partial A$ can be expressed as $\xi=\xi_{0}(\eta)$. The nature of these operators $(\mathscr{L} q)_{\eta}$ and $(\mathscr{K} q)_{\eta}$ will be stated as Propositions 1,2 and proved by using a theorem in [12] together with some elementary calculations.

Lemma 4. The variation $q=I_{0}(d \xi / d t)$ along $\xi_{0}(\eta)$ corresponding to the 1-parameter "group" of area-preserving diffeomorphisms generated by $(d x / d t)=\phi_{y},(d y / d t)=-\phi_{x}$ is given by $q=\phi_{\eta}$.

Proof. $(d \xi / d t)=\xi_{x}(d x / d t)+\xi_{y}(d y / d t) . x_{\xi}=I \eta_{y}, x_{\eta}=-I \xi_{y}, y_{\xi}=-I \eta_{x}$, and $y_{\eta}=I \xi_{x}$ hold in general. Thus, $q=\mathrm{I} \xi_{x}(d x / d t)+\mathrm{I} \xi_{y}(d y / d t)=y_{\eta} \dot{x}-x_{\eta} \dot{y}=y_{\eta} \phi_{y}+\phi_{x} x_{\eta}=\phi_{\eta}$.

Corollary 1. The symplectic structure $\left(\psi_{1}, \psi_{2}\right) \rightarrow \int_{\partial A} \psi_{1} d \psi_{2}$ in [20] is the same as ours $\left(f_{\eta}, g_{\eta}\right) \rightarrow \int_{\partial A} f g_{\eta} d \eta$ by taking $f=\psi_{1}, g=\psi_{2}$ on $\xi_{0}(\eta)$. For translations in $x$-direction, $y$-direction, and rotations in the plane, we take $\phi=y, \phi=-x$, and $\phi=\frac{1}{2}\left(x^{2}+y^{2}\right)$ respectively.

Corollary 2. $(\alpha)-\psi_{x}=\int G y_{\eta^{\prime}} d \eta^{\prime}$,

$$
\begin{aligned}
& \text { (B) } \psi_{y}=\int G x_{\eta^{\prime}} d \eta^{\prime} \text {, } \\
& (\gamma)-y \psi_{x}+x \psi_{y}=\int \frac{1}{2} G\left(x^{2}+y^{2}\right)_{\eta^{\prime}} d \eta^{\prime} .
\end{aligned}
$$

Lemma 5. For a rotating vortex patch $\chi_{A}$,

( $\alpha) \mathscr{L} x_{\eta}=-\Omega y$, 
(B) $\mathscr{L} y_{\eta}=\Omega x$,

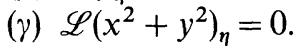

Proof. To see $(\alpha), \quad x_{\eta}=\left(\hat{\psi}_{\xi} / I\right) x_{\eta}+\psi_{y} \quad$ (for $\left.\quad \psi_{y}=\int G x_{\eta^{\prime}} d \eta^{\prime}\right)=-\xi_{y} \hat{\psi}_{\xi}+\psi_{y} \quad$ (for $\left.x_{\eta}=-\xi_{y} I\right)=-\hat{\psi}_{y}+\psi_{y}\left(\right.$ for $\left.\hat{\psi}_{\eta}=0\right)=-\psi_{y}-\Omega y+\psi_{y}=-\Omega y$.

Similarly one has $(\beta)$ and $(\gamma)$.

Let $\mathscr{A}$ be a linear operator on a complex Hilbert space $\mathscr{H}$. Set $R_{\lambda}=$ $R_{\lambda}(\mathscr{A})=\left\{u \in \mathscr{H} \mid(\mathscr{A}-\lambda)^{n} u=0\right.$ for some positive integer $\left.n\right\}$. For an eigenvalue $\lambda$ (i.e. $\left.R_{\lambda} \neq\{0\}\right), R_{\lambda}$ is called the associated root subspace of $\mathscr{A}$, when the dimension of $R_{\lambda}$ is finite. Now, we recall a theorem from Gohberg and Krein [12].

Theorem. Suppose that (1) $L$ is a self-adjoint operator with a discrete spectrum, (2) $L^{-1}$ exists and $T L^{-1}$ is a Hilbert-Schmidt operator. Then, $L+T$ has a discrete spectrum, and the system of its root subspaces is complete in $\mathscr{H}$.

Proposition 1. The operator $(\mathscr{L} q)_{\eta}$ has a discrete spectrum $\Sigma\left(\mathscr{L}_{\eta}\right)$. Its system of root subspaces is complete in $\llbracket Q \rrbracket=Q \oplus i Q$.

Proof. Express $(\mathscr{L} q)_{\eta}$ as $\left(\hat{\psi}_{\xi} q\right)_{\eta}-\int G q_{\eta^{\prime}} d \eta^{\prime}$. Thus, one can apply the above theorem to $i(\mathscr{L} q)_{\eta}=i\left(\hat{\psi}_{\xi} q\right)_{\eta}-i \int G q_{\eta^{\prime}} d \eta^{\prime}$ provided we use an (equivalent) inner product with weight $-\hat{\psi}_{\xi}(>0)$ on $Q \oplus i Q$.

Fix a cross-section $\pi_{\alpha}$ with $\int \alpha\left(x^{2}+y^{2}\right)_{\eta}=1, \int \alpha x_{\eta}=\int a y_{\eta}=0$. Given a set $S$, [S] and $\llbracket S \rrbracket$ denote the linear span of $S$ with real and complex coefficients respectively. One can readily see:

Lemma 6. $Q=\left[x_{\eta}, y_{\eta}\right] \oplus\left[\alpha_{\eta},\left(x^{2}+y^{2}\right)_{\eta}\right] \oplus \mathscr{N}$ is a decomposition into symplectic subspaces.

Recall that $(\mathscr{K} q)_{\eta}$ is the induced transformation of $(\mathscr{L} q)_{\eta}$ on $\mathscr{N}$.

Proposition 2. The linear operator $\mathscr{K}_{\eta}$ possesses a discrete spectrum $\Sigma\left(\mathscr{K}_{\eta}\right)$. $\Sigma\left(\mathscr{L}_{\eta}\right)=\{0,0, \pm i \Omega\} \cup \Sigma\left(\mathscr{K}_{\eta}\right)$ (including algebraic multiplicities). Furthermore, the system of root subspaces of $\mathscr{K}_{\eta}$ is complete in $\llbracket \mathscr{N} \rrbracket=\mathscr{N} \oplus i \mathcal{N}$.

Proof. The matrix representation of $\mathscr{L}_{\eta}$ with respect to the decomposition given in Lemma 6 has the form:

$$
\left(\begin{array}{rr|cc|c}
0 & \Omega & 0 & 0 & 0 \\
-\Omega & 0 & 0 & 0 & 0 \\
\hline 0 & 0 & 0 & 0 & 0 \\
0 & 0 & d & 0 & \times \\
\hline 0 & 0 & \times & 0 & \mathscr{K}_{\eta}
\end{array}\right) \text { with } d=-\int \alpha_{\eta} \cdot \mathscr{L}\left(\alpha_{\eta}\right)=-2 \hat{E}_{2}\left(\alpha_{\eta}\right) .
$$

Therefore, $\quad$ (i) $\quad R_{ \pm i \Omega}\left(\mathscr{L}_{\eta}\right)=\llbracket x_{\eta} \pm i y_{\eta} \rrbracket \oplus R_{ \pm i \Omega}^{*}, \quad$ with $\quad R_{ \pm \Omega \Omega}^{*}=R_{i \Omega}\left(\mathscr{L}_{\eta} \mid\left[\alpha_{\eta}\right.\right.$, $\left.\left.\left(x^{2}+y^{2}\right)_{\eta}\right] \oplus \mathscr{N}\right) \cdot R_{0}\left(\mathscr{L}_{\eta}\right)=\llbracket \alpha_{\eta}+n,\left(x^{2}+y^{2}\right)_{\eta} \rrbracket \oplus R_{0}\left(\mathscr{K}_{\eta}\right)$ for some $n \in \mathscr{N}$.

(ii) $R_{ \pm \pm \Omega}^{*} \leqq \llbracket\left(x^{2}+y^{2}\right)_{\eta}, \mathscr{N} \rrbracket$ and $R_{\lambda}\left(\mathscr{L}_{\eta}\right) \leqq \llbracket\left(x^{2}+y^{2}\right)_{\eta^{\prime}} \cdot \mathcal{N} \rrbracket$ for $\lambda \neq 0, \pm i \Omega$.

(iii) $R_{ \pm \Omega \Omega}^{*} \approx R_{ \pm \Omega \Omega}\left(\mathscr{K}_{\eta}\right)$, and $R_{\lambda}\left(\mathscr{L}_{\eta}\right) \approx R_{\lambda}\left(\mathscr{K}_{\eta}\right)$ for $\lambda \neq 0$, $\pm i \Omega$, via the projection $\llbracket\left(x^{2}+y^{2}\right)_{\eta}, \mathcal{N} \rrbracket \rightarrow \llbracket \mathscr{N} \rrbracket$.

Our Proposition follows from the above three facts (i), (ii) and (iii).

\section{(B) The Smooth Dependence of $E\left(\chi_{B}\right)$ on the Boundary $\partial B$}

As before, let $(\xi, \eta)$ be a smooth coordinate system $x=x(\xi, \eta), y=y(\xi, \eta)$, near a $C^{1}$ boundary of a vortex patch $\chi_{A}$ so that $\partial A$ can be expressed as $\xi=\xi_{0}(\eta)$. Denote by 
$C^{1}(\eta), C^{0}(\eta)$ the Banach spaces of $C^{1}, C^{0}$ functions in $\eta$. Take $\mathfrak{A} \subset C^{1}(\eta)$ an open neighborhood of $\chi_{A}$ in $C^{1}(\eta)$, so that each $\xi \in \mathfrak{A}$ represents a vortex patch $\chi_{\xi}$ near $\chi_{A}$ with boundary given by $\xi=\xi(\eta)$. To each $\xi \in \mathfrak{A}$, let $\psi(\xi), \psi_{x}(\xi), \psi_{y}(\xi)$ be the restrictions of $\psi\left(\chi_{\xi}\right), \psi_{x}\left(\chi_{\xi}\right), \psi_{y}\left(\chi_{\xi}\right)$ on the boundary $\xi=\xi(\eta)$ respectively. Thus, $\psi(\xi) \in C^{1}(\eta)$ and $\psi_{x}(\xi), \psi_{y}(\xi) \in C^{0}(\eta)$. Notice that $\psi$ is usually not even $C^{2}$ on the variables $x, y$. Amazingly, one still can have the next smoothness result.

Proposition 3. (a) The map $\boldsymbol{\xi} \in \mathfrak{A} \rightarrow \psi(\xi) \in C^{1}(\eta)$ is smooth.

(b) The energy function $E\left(\chi_{\xi}\right)$ is smooth in $\xi$.

For $(\delta E / \delta \xi)=I_{0} \psi(\xi)$, it suffices to prove (a).

Now we need the following facts:

( $\alpha$ ) Suppose $\mathscr{A}(\xi ; \eta), \mathscr{B}(\xi ; \eta)$ are smooth in $\xi \in \mathfrak{A}, C^{0}$ in $\eta$. Then so is the product $\mathscr{A} \cdot \mathscr{B}$, the quotient $\mathscr{A} / \mathscr{B}$ (if $\mathscr{B} \neq 0$ ).

( $\beta$ ) Suppose $\mathscr{A}\left(\xi ; \eta, \eta^{\prime}\right)$ is smooth in $\xi, C^{0}$ in $\eta, \eta^{\prime}$. Then $\int \mathscr{A}\left(\xi ; \eta, \eta^{\prime}\right) d \eta^{\prime}$ is smooth in $\xi$, $C^{0}$ in $\eta$.

( $\gamma)$ Let $F(\xi, \eta) \in R$ be smooth. Then, $F(\xi(\eta), \eta) \in C^{1}(\eta)$ and the map $\xi \rightarrow F(\xi(\eta), \eta) \in C^{1}$ is smooth.

( $\delta$ ) Write $x(\eta)=x(\xi(\eta), \eta)$. For $\eta-\eta^{\prime}$ small, $x(\eta)-x\left(\eta^{\prime}\right)=g\left(\xi ; \eta, \eta^{\prime}\right)\left(\eta-\eta^{\prime}\right)$ with $g\left(\xi ; \eta, \eta^{\prime}\right)=\int_{0}^{1}(d x / d \eta)\left(t \eta+(1-t) \eta^{\prime}\right) d t$. Thus, $g\left(\xi ; \eta, \eta^{\prime}\right)$ is $C^{\infty}$ in $\xi$ and $C^{0}$ in $\eta, \eta^{\prime}$. The same kind of result holds for $y(\eta)$ with $y\left(\eta^{\prime}\right)-y\left(\eta^{\prime}\right)=h\left(\xi ; \eta, \eta^{\prime}\right)\left(\eta-\eta^{\prime}\right)$, and $g^{2}+h^{2} \neq 0$.

Proof. It suffices to show that the maps

$$
\xi \rightarrow \psi(\xi), \psi_{x}(\xi), \psi_{y}(\xi) \in C^{0}(\eta) \text { are smooth. }
$$

(1) Write $\psi=(1 / 4 \pi)\left\{\frac{1}{2}\left(\int\left(x^{\prime}-x\right) \ln \left(1 / r^{2}\right) d y^{\prime}-\int\left(y^{\prime}-y\right) \ln \left(1 / r^{2}\right) d x^{\prime}\right)+\int d x^{\prime} d y^{\prime}\right\}$, where $r^{2}=\left(x-x^{\prime}\right)^{2}+\left(y-y^{\prime}\right)^{2}$.

Thus,

$$
\begin{aligned}
\psi= & \frac{1}{4 \pi}\left\{\frac { 1 } { 2 } \left(\int\left(x^{\prime}-x\right) \ln \frac{1}{r^{2}} \frac{d y^{\prime}}{d \eta^{\prime}} d \eta^{\prime}\right.\right. \\
& \left.\left.-\int\left(y^{\prime}-y\right) \ln \frac{1}{r^{2}} \cdot \frac{d x^{\prime}}{d \eta^{\prime}} d \eta^{\prime}\right)+\int \frac{\partial\left(x^{\prime}, y^{\prime}\right)}{\partial\left(\xi^{\prime}, \eta^{\prime}\right)} d \xi^{\prime} d \eta^{\prime}\right\} .
\end{aligned}
$$

From facts $(\alpha), \quad(\gamma), \quad(\delta), \quad\left(x^{\prime}-x\right) \ln \left(1 / r^{2}\right), \quad\left(y^{\prime}-y\right) \ln \left(1 / r^{2}\right), \quad\left(d y^{\prime} / d \eta^{\prime}\right), \quad\left(d x^{\prime} / d \eta^{\prime}\right)$, $\left(\partial\left(x^{\prime}, y^{\prime}\right) /\left(\partial\left(\xi^{\prime}, \eta^{\prime}\right)\right)\right.$ are $C^{\infty}$ in $\xi$ and $C^{0}$ in $\eta, \eta^{\prime}$. By the fact $(\beta) \psi$ is $C^{\infty}$ in $\xi$ and $C^{0}$ in $\eta$,

(2) $\psi_{y}=\frac{1}{4 \pi} \int \ln \frac{1}{r^{2}} d x^{\prime}=\frac{1}{4 \pi} \int \ln \frac{1}{r^{2}} d\left(x^{\prime}-x\right)$

$$
=\frac{1}{4 \pi} \int\left(x^{\prime}-x\right) d\left(\ln r^{2}\right)=\frac{1}{2 \pi} \int\left[\frac{\left(x^{\prime}-x\right)^{2} d x^{\prime}}{r^{2}} \frac{\left(x^{\prime}-x\right)\left(y^{\prime}-y\right)}{d \eta^{\prime}}+\frac{d y^{\prime}}{d \eta^{\prime}}\right] d \eta^{\prime} .
$$

From facts $(\alpha),(\gamma),(\delta)\left(\left(x^{\prime}-x\right)^{2} / r^{2}\right),\left(\left(x^{\prime}-x\right)\left(y^{\prime}-y\right) / r^{2}\right),\left(d x^{\prime} / d \eta^{\prime}\right),\left(d y^{\prime} / d \eta^{\prime}\right)$ are $C^{\infty}$ in $\xi$ and $C^{0}$ in $\eta, \eta^{\prime}$. By the fact $(\beta), \psi_{y}$ is smooth in $\xi$ and $C^{0}$ in $\eta$.

(3) Similarly, one gets $\psi_{x}=-(1 / 4 \pi) \int \ln \left(1 / r^{2}\right) d y^{\prime}$ is also smooth in $\xi$ and $C^{0}$ in $\eta$. Combining statements (1), (2) and (3) above we get the smoothness of the map $\xi \rightarrow \psi(\xi)$. 


\section{(C) The Stability of Thomson's $m$ Point Vortices}

Suppose we have a vortex patch consisting of $m$ disk-like components with small radius $\varepsilon$ and centered at $z_{0}, \ldots, z_{m-1}$ in a rotating system with an angular velocity $\Omega$. The velocities at points $z_{i}=x_{j}+i y_{j}$ are approximately given by

$$
\begin{aligned}
& \left\{\begin{array}{l}
\frac{d x_{j}}{d t}=\frac{\partial \hat{H}}{\partial y_{j}} \\
\frac{d y_{j}}{d t}=-\frac{\partial \hat{H}}{\partial x_{j}},
\end{array} \text { or } \quad \frac{d z_{j}}{d t}=-2 i \frac{\partial \hat{H}}{\partial \bar{z}_{j}}, \quad j=0,1, \ldots, m-1 .\right. \\
& \text { with } \hat{H}\left(z_{1}, \ldots, z_{m}\right)=\frac{\varepsilon^{2}}{4} \sum_{j \neq l} \ln \frac{1}{\left|z_{j}-z_{l}\right|}+\frac{\Omega}{2} \sum_{j} z_{j} \bar{z}_{j} .
\end{aligned}
$$

(For $\hat{\psi}=\hat{H}+$ constant $(\varepsilon)$.) For the sake of completeness, we include the studies of the stability of this Hamiltonian system in a complex variable setting.

It is elementary to establish the following

Lemma 7. $\ln (1 /|1+t z|)=-t(z+\bar{z}) / 2+\left(t^{2} / 4\right)\left(z^{2}+\bar{z}^{2}\right)+\cdots$ for small $t$.

Lemma 8. Let $2 \beta_{1}=\sum_{k=1}^{m-1} 1 /\left(1-\tau^{k}\right)$. Then $2 \beta_{1}=(m-1 / 2)$.

Lemma 9. Let $2 \beta_{2}=\sum_{k=1}^{m-1} 1 /\left(1-\tau^{k}\right)^{2}$. Then $2 \beta_{2}=-(m-1)(m-5) / 12$.

Lemma 10. Let $s_{p}=\sum_{k=1}^{m-1}\left(\tau^{k p} /\left(1-\tau^{k}\right)^{2}\right)(1 \leqq p \leqq m)$. Then $\quad s_{p}=\frac{1}{2}(p-2)(m-p)$ $-((m-1)(m-5) / 12)$.

Recall that $\tau=e^{i 2 \pi / m}$, and let $z_{j}=\tau^{j}\left(1+\zeta_{j}\right)$, for each $j$. Using Lemmas 7,8, one can establish that $z_{j}^{0}=\tau^{j}, j=0, \ldots, m-1$, is a stationary solution of $\left(d z_{j} / d t\right)=$ $-2 i\left(\partial \hat{H} / \partial \bar{z}_{j}\right)$, with an angular velocity $\Omega_{0}=\beta_{1} \varepsilon^{2}(=(m-1) / 4) \varepsilon^{2}$. Furthermore (use also Lemma 9),

$$
\begin{aligned}
\hat{H} & =\hat{H}\left(\tau^{0}, \ldots, \tau^{m-1}\right)+\frac{1}{2}\left[\frac{1}{4} \sum_{j \neq l} \operatorname{Re}\left(\frac{\tau^{j} \zeta_{j}-\tau^{l} \zeta_{l}}{\tau^{j}-\tau^{l}}\right)^{2}+\frac{m-1}{4} \sum_{j} \zeta_{j} \bar{\zeta}_{j}\right]+0\left(|\zeta|^{3}\right) \\
& =\hat{H}\left(\tau^{0}, \ldots, \tau^{m-1}\right)+\frac{1}{2}\langle\zeta, L \zeta\rangle+0\left(|\zeta|^{3}\right) .
\end{aligned}
$$

Here, $L \zeta=\beta_{1} \zeta+B \bar{\zeta}$ is symmetric with respect to $\langle\zeta, \eta\rangle=\operatorname{Re} \zeta \bar{\eta}$ with

$$
B=\left(b_{l j}\right), \quad b_{l j}=\left\{\begin{array}{l}
\beta_{2}, l=j \\
-\frac{1}{2} \frac{\tau^{l} \tau^{j}}{\left(\tau^{i}-\tau^{j}\right)^{2}} \text { a real number, } l \neq j .
\end{array}\right.
$$

Now set $\quad \gamma^{p}=\left(\begin{array}{c}1 \\ \tau^{p} \\ \vdots \\ \left(\tau^{p}\right)^{m-1}\end{array}\right), \quad p=0,1, \ldots, m-1$.

Lemma 11. $B \bar{\gamma}^{p}=\left(-\frac{1}{2} s_{p+1}+\beta_{2}\right) \bar{\gamma}^{p}$.

Proof. $\left[B \bar{\gamma}^{p}\right]_{l}=\sum_{j} b_{l j} \tau^{-p j}=\sum_{j \neq l}-\frac{1}{2} \frac{\tau^{l} \tau^{j} \tau^{-p j}}{\left(\tau^{l}-\tau^{j}\right)^{2}}+\beta_{2} \tau^{-p l}$ 


$$
\begin{aligned}
& =-\frac{1}{2} \sum_{k=l-j \neq 0} \frac{\tau^{l} \tau^{l-k} \tau^{-p l} \tau^{p k}}{\left(\tau^{l}-\tau^{l-k}\right)^{2}}+\beta_{2} \tau^{-p l} \\
& =-\frac{1}{2}\left(\sum_{k \neq 0} \frac{\tau^{(p+1) k}}{\left(\tau^{k}-1\right)^{2}}\right) \tau^{-p l}+\beta_{2} \tau^{-p l} .
\end{aligned}
$$

Corollary 3. The linear transformation $L$ has eigenvalues $(m-1) / 4 \mp(1 / 4)(p-1)$ $(m-p-1)$ with eigenvectors $\operatorname{Re} \gamma^{p}, \operatorname{Im} \gamma^{p} ; i \operatorname{Re} \gamma^{p}, i \operatorname{Im} \gamma^{p}, p=0,1, \ldots, m-1$ except the zero vectors $\operatorname{Im} \gamma^{0}, i \operatorname{Im} \gamma^{0}, \operatorname{Im} \gamma^{m / 2}, i \operatorname{Im} \gamma^{m / 2}$ (if $m$ is even). that

Let $e=(1, \ldots, 1)$. Through simple computations, we can immediately deduce

Proposition 4. $L \mid[e, i e]^{\perp}$ is

(a) positive definite for $2 \leqq m \leqq 6$,

(b) positive semi-definite for $m=7$,

(c) of saddle type for $m \geqq 8$.

Corollary 4. The system $\left(d z_{j} / d t\right)=-2 i\left(\partial \hat{H} / \partial \bar{z}_{j}\right), j=0,1, \ldots, m-1$ is nonlinearly stable for $m \leqq 6$ and is neutrally stable for $m \leqq 7$.

There are redundant in our expressions for eigenvalues of $L ; \operatorname{Re} \gamma^{p}=\operatorname{Re} \gamma^{p \prime}$, $\operatorname{Im} \gamma^{p}=-\operatorname{Im} \gamma^{p^{\prime}}$ for $p+p^{\prime}=m$. Thus, we can obtain an orthogonal decomposition by avoiding such repetitions:

$$
[e, i e]^{\perp}=\sum_{1 \leqq p<m / 2}\left[\operatorname{Re} \gamma^{p}, \operatorname{Im} \gamma^{p}\right] \oplus\left[i \operatorname{Re} \gamma^{p}, i \operatorname{Im} \gamma^{p}\right] \oplus \sum_{p=m / 2}\left[\operatorname{Re} \gamma^{p}, i \operatorname{Re} \gamma^{p}\right] .
$$

$L$ has index 1 on $\left[\operatorname{Re} \gamma^{p}, i \operatorname{Re} \gamma^{p}\right] 2 p=m$, and even index $(0$ or 2$)$ on other subspaces. Hence, we have

Proposition 5. For $m \geqq 8, L \mid[e, i e]^{\perp}$ is of even index if and only if $m$ is odd.

Finally, we linearize the system in $\zeta$-coordinates; $\left(d \zeta_{j} / d t\right)=-i \varepsilon^{2}(L \zeta)_{j}, j$ $=0,1, \ldots, m-1$. This is a Hamiltonian system with a quadratic Hamiltonian $\hat{H}_{2}$ with respect to the symplectic structure $\omega\left(\zeta, \zeta^{\prime}\right)=\left\langle i \zeta, \zeta^{\prime}\right\rangle$. One has a symplectic decomposition of $-i(L \zeta)$ into eigenspaces;

$$
[e, i e]^{\perp}=\sum_{1 \leqq p<m / 2}\left[\operatorname{Re} \gamma^{p}, i \operatorname{Re} \gamma^{p}\right] \oplus\left[\operatorname{Im} \gamma^{p}, i \operatorname{Im} \gamma^{p}\right] \oplus \sum_{p=m / 2}\left[\operatorname{Re} \gamma^{p}, i \operatorname{Re} \gamma^{p}\right]
$$

For an even $m=2 s,-i(L \zeta) \mid\left[\operatorname{Re} \gamma^{s}, i \operatorname{Re} \gamma^{s}\right]$ has eigenvalues $\pm \frac{1}{4} \sqrt{(s-1)^{4}-(2 s-1)^{2}}$. For an odd $m=2 s+1, \quad-i(L \zeta) \mid\left[\operatorname{Re} \gamma^{s}, i \operatorname{Re} \gamma^{s}\right] \quad$ has eigenvalues $\pm \frac{1}{4} \sqrt{(s-1)^{2} s^{2}-(2 s)^{2}}$. Thus, we obtain

Proposition 6. For $m \geqq 8$, (a) $-i(L \zeta) \mid[e, i e]^{\perp}$ has a pair of real eigenvalues $\pm \lambda, \lambda>0$. (b) The system $\left(d z_{j} / d t\right)=-2 i\left(\partial \hat{H} / \partial \bar{z}_{j}\right)$ is not neutrally stable.

\section{References}

1. Abraham, R., Marsden,: Foundation of mechanics. Second edition Reading, MA: Addison-Wesley 1978

2. Aref, H.: Integrable, chaotic and turbulent vortex motion in two-dimensional flows. Ann. Rev. Fluid Mech. 15 (1983) 
3. Arnol'd, V. I.: Conditions for nonlinear stability of stationary plane curvilinear flows of an ideal fluid. Sov. Math. Dokl. 6, 773-777 (1965)

4. Arnol'd, V. I.: On an a priori estimate in the theory of hydrodynamical stability. Trans. Am. Math. Soc. 79, 267-269 (1969)

5. Benjamin, T. B.: The alliance of practical and analytic insights into the nonlinear problems of fluid mechanics. Applications of methods of functional analysis to problems of mechanics. Lecture Notes in Mathematics, No. 503, Berlin, Heidelberg, New York 1976, pp. 8-29

6. Benjamin, T. B.: Impulse, flow force and variational principles. IMA J. Appl. Math. 32, 3-68 (1984)

7. Burbea, J., Landau, M.: The Kelvin waves in vortex dynamics and their stability. J. Comput. Phys. 45, $127-156(1982)$

8. Deem, G. S., Zabusky, N. J.: Vortex waves: stationary 'V-states', interactions, recurrence, and breaking. Phy. Rev. Lett. 40, 859-62 (1978)

9. Dritschel, D. G.: Steady state structures of co-rotating uniform vortices (preprint). Princeton University, 1984

10. Dritschel, D. G.: The stability and energetics of co-rotating uniform vortices (preprint), 1984

11. Dritschel, D. G.: The nonlinear stability of co-rotating uniform vortices (preprint), 1984

12. Gohberg, I. C., Krein, M. G.: Introduction to the theory of linear nonselfadjoint operators. Transl. Math. Monogr. 18, (1969)

13. Gromoll, D., Meyer, W.: On differentiable functions with isolated critical points. Topology 8, 361369 (1969)

14. Holm, D., Marsden, J., Ratiu, T., Weinstein, A.: Nonlinear stability of fluid and plasma equilibria. Phys. Rep. 123, 1-116 (1985)

15. Kelvin, Lord: On the stability of steady and of periodic fluid motion. Philes Mag. (5) 23, 529-39 (1887)

16. Krein, M. G.: A generalization of several investigations of A. M. Liapunov on linear differential equations with periodic coefficients. Dokl. Akad. Nauk. SSSR 73, 445-448

17. Lamb, H.: Hydrodynamics. New York: Dover publications 1945

18. Love, A. E. H.: On the stability of certain vortex motion. Proc. Soc. Lond., 18-42 (1983)

19. Marchioro, C., Pulvirenti, M.: Some considerations on the nonlinear stability of stationary planar Euler flows. Commun. Math Phys. 100, 343-354 (1985)

20 Marsden, J., Weinstein, A.: Co-adjoint orbits, vortices, and Clebsch variables for incompressible fluids. Physica 7D, 305-323 (1983)

21. McKee, T. G.: Existence and structure on non-circular stationary vortices. Thesis, Brown University, 1981

22. Meiron, D. I., Saffman, P. G., Schatzman, J. C.: The stability of inviscid vortex streets of finite cored vortices (preprint) C.I.T. 1983

23. Norbury, J.: A family of steady rings. J. Fluid Mech. 57, 417-431 (1973)

24. Pierrehumbert, R. T.: A family of steady translating vortex pairs with distributed vorticity. J. Fluid Mech. 99, 129-144 (1980)

25. Saffman, P. G., Szeto, R.: Equilibrium shapes of a pair of equal uniform vortices. Phys. Fluids 23(12), - (1980)

26. Tang, Y.: Nonlinear stability of vortex patches, Thesis, 1984, S.U.N.Y. at Buffalo

27. Turkington, B.: Co-rotating steady vortex flows with $N$-fold symmetry. Nonlinear Anal. (to appear) 1984

28. Turkington, B.: On steady vortex flows in two dimensions, I. Commun. Partial Differ. Equations, 999-1030 (1983)

29 Wan, Y.H.: Variational principles for Hill spherical vortex and nearly spherical vortices, preprint, S.U.N.Y. at Buffalo, 1985

30. Wan, Y.H., Pulvirenti, M.: Nonlinear stability of circular vortex patches. Commun. Math. Phys. 99, 435-450 (1985)

31. Weinstein, A.: Stability of Poisson-Hamilton equilibria. Fluids and Plasmas, Contemporary Math. Vol. 28, 1984

32. Williamson, J.: On the algebraic problem concerning the normal forms of linear dynamical systems. Am. J. Math. 58, 141-163 (1936) 
33. Wu, H., Overman, E., Zabusky, N.: Steady-state solutions of the Euler equations in two dimensions: Rotating and translating $V$-states with limiting cases. I. Numerical Results. J. Comput. Phys. , 54 (1984)

34. Zubusky, N. J.: Recent developments in contour dynamics for the Euler equations. Ann. New York Acad. Sci. 373, 160-170 (1981)

Communicated by J. L. Lebowitz

Received October 31, 1985; in revised form March 18, 1986 